\title{
APPROXIMATE CONTROLLABILITY OF IMPULSIVE STOCHASTIC FRACTIONAL DIFFERENTIAL EQUATIONS WITH NONLOCAL CONDITIONS
}

\author{
ALKA CHADHA ${ }^{1}$, S.N. BORA ${ }^{2}$, R. SAKTHIVEL ${ }^{3}$ \\ ${ }^{1,2}$ Department of Mathematics \\ Indian Institute of Technology Guwahati \\ Guwahati, 781039, INDIA \\ ${ }^{3}$ Department of Mathematics \\ Sungkyunkwan University \\ Suwon, 440-746, SOUTH KOREA
}

\begin{abstract}
This paper studies the approximate controllability of an impulsive neutral stochastic integro-differential equation with nonlocal conditions and infinite delay involving the Caputo fractional derivative of order $q \in(1,2)$ in separable Hilbert space. The existence of the mild solution to fractional stochastic system with nonlocal and impulsive conditions is first proved utilizing fixed point theorem, stochastic analysis, fractional calculus and solution operator theory. Then, a new set of sufficient conditions proving approximate controllability of nonlocal semilinear fractional stochastic system involving impulsive effects is derived by assuming the associated linear system is approximately controllable. Illustrating the obtained abstract results, an example is considered at the end of the paper.
\end{abstract}

AMS Subject Classification: 34K37, 34K30, 35R11, 47N20, 60H15

Key Words: approximate controllability, fixed point theorem, Caputo derivative, stochastic integro-differential equation, nonlocal conditions, resolvent operator

Received: February 3, 2017; Accepted: September 10, 2017;

Published: January 4, $2018 \quad$ doi: $10.12732 /$ dsa.v27i1.1

Dynamic Publishers, Inc., Acad. Publishers, Ltd. https://acadsol.eu/dsa

\section{INTRODUCTION}

Recently, differential equations involving fractional derivative have gained considerable popularity and importance, mainly due to its demonstrated applications in numerous diverse and widespread fields in science and engineering. Fractional calculus 
has been successfully applied to problems in systems biology, physics, chemistry and biochemistry, hydrology, medicine, and finance. In many cases, these new fractionalorder models are more adequate than the previously used integer-order models, because fractional derivatives and integrals enable the description of the memory and hereditary properties inherent in various materials and processes that are governed by anomalous diffusion. The fractional viscoelastic model, that is the linear viscoelastic model involving fractional order operators in the constitutive equations, is capable of describing the behavior of various viscoelastic materials utilizing a few parameters. Hence, there is a growing need to find the solution behavior of these fractional differential equations. For more details, we refer to the monographs [2], [3] and papers $[25,27,37,38,40,43,44]$. In addition, neutral differential equations of integer or fractional order arise in various areas of real world problems which play an important role in the theory of functional differential equations, and receive much attention in the last few decades. Such equations find many applications in natural sciences and technology, for example, a study of heat conduction in materials with memory, but as a rule, they have specific properties making their study difficult both in the aspects of ideas and techniques. For more details, see [15, 19, 22, 29, 39, 43, 44, 45, 47, 48] and references cited therein. For the study of differential equations with nonlocal initial conditions, we refer to the papers [11, 12, 17, 19, 20, 36, 37, 39, 40, 42, 44, 49, 50, 52].

On the other hand, stochastic differential equations play a prominent role in a range of application areas, including biology, chemistry, epidemiology, mechanics, microelectronics, economics and finance. Some of the typical applications of nonlinear stochastic differential equations are the vibration of tall building and bridges under the action of wind or earthquake, vehicles moving on rough roads, ships and offshore oil platform subjected to wind and ocean waves, price processes in financial markets and electronic circuits subjected to thermal noise. For more study of stochastic differential equations and their applications, we refer to the monographs $[8,9,10]$. Recently, existence, uniqueness and stability results for stochastic differential equations have been studied in $[14,15,16,19,22,28,41,42,43,45]$. In recent years, many systems in physics and biology exhibit impulsive dynamical behavior because of sudden jumps at certain instants in the evolution process. A lot of dynamic systems have variable structures subjects to stochastic abrupt changes resulting from abrupt phenomena, for example, stochastic failure and repair of components, changes in the interconnections of subsystems, sudden environmental changes and so on. For some recent works on the existence results of impulsive stochastic differential equations, we refer the reader to monographs $[6,7]$ and $[14,22,23,28,39,42,43]$.

The study of controllability plays an important role in the control theory and engineering. The problem of controllability of various kinds of differential, integrodifferential equations and impulsive differential equations are studied, see. The ap- 
proximate controllability is the weaker concept of controllability receiving much attention. In this case it is possible to steer the system to an arbitrary small neighborhood of the final state $[17,18,20,21,24,32,33,35,50,51]$. However, stochastic control theory which is a generalization of classical control theory has rarely been reported. As a matter of fact, the accurate analysis or assessment subjected to a realistic environment has to take into account the potential randomness in the system properties, such as fluctuations in the stock market or noise in a communication network. All these problems in mathematics are modeled and described by stochastic differential equations or stochastic integro-differential equations with delay and impulse. The biggest difficulty is the analysis of a stochastic control system and stochastic calculations induced by the stochastic process. For more details, see [14, 16, 19, 23, 34, 36, 39, 50, 52].

In this paper, we study the the following integro-differential equation with infinite delay involving nonlocal and impulsive conditions in a separable Hilbert space $(E, \|$. $\|,<\cdot, \cdot>)$

$$
\begin{aligned}
& { }^{C} D_{t}^{q}\left[u(t)+\int_{0}^{t}(t-s) G\left(s, u_{s}, \int_{0}^{s} a_{1}\left(s, \tau, u_{\tau}\right) d \tau\right) d s\right]=A u(t)+B x(t) \\
& +F\left(t, u_{t}, \int_{0}^{t} a_{2}\left(t, s, u_{s}\right) d s\right)+H\left(t, u_{t}, \int_{0}^{t} a_{3}\left(t, s, u_{s}\right) d s\right) \frac{d W(t)}{d t}, t \in[0, T],(1, \\
& \Delta u\left(t_{i}\right)=I_{i}\left(u\left(t_{i}\right)\right), \Delta u^{\prime}\left(t_{i}\right)=J_{i}\left(u\left(t_{i}\right)\right) i=1,2, \cdots, m, m \in \mathbb{N} \\
& u(0)+g(u)=u_{0}=\phi \in \mathfrak{B}_{v}, u^{\prime}(0)+h(u)=u_{1} \in E
\end{aligned}
$$

where $1<q<2,{ }^{C} D_{t}^{q}$ is the generalized fractional derivative in Caputo sense, $A$ : $D(A) \subset E \rightarrow E$ is a closed and linear operator with the domain $D(A)$ defined in a Hilbert space $E, t_{i}(i=1, \cdots, m)$ are the fixed number such that $0=t_{0}<t_{1}<\cdots<$ $t_{m}=T$, and $\left.\Delta u\right|_{t=t_{k}}=u\left(t_{k}^{+}\right)-u\left(t_{k}^{-}\right)$, where $u\left(t_{k}^{+}\right)$and $u\left(t_{k}^{-}\right)$denote the right and left limits of $u(t)$ at $t=t_{k}$, respectively. The $W(t)$ denotes the $K$-valued Wiener process with a finite trace nuclear covariance operator $\mathcal{Q}$. The control function $x(\cdot)$ takes the values $L^{2}([0, T], \mathbb{U})$, where $\mathbb{U}$ is a Hilbert space and $B$ is a bounded linear operator from $\mathbb{U}$ into $E$. The history $u_{t}:(-\infty, 0] \rightarrow E, u_{t}(\theta)=u(t+\theta), \theta \leq 0$ belongs to an abstract phase space $\mathfrak{B}_{v}$ and the initial function $\phi=\{\phi(t): t \in$ $(-\infty, 0]\}$ is a $\mathcal{F}_{0}$-measurable, $\mathfrak{B}_{v}$ random variable independent of Wiener process $W(t)$ with finite second moments. The nonlinear functions $G, F:[0, T] \times \mathfrak{B}_{v} \times E \rightarrow E$, $H:[0, T] \times \mathfrak{B}_{v} \times E \rightarrow L(K, E), a_{1}, a_{2}, a_{3}: D_{1} \times \mathfrak{B}_{v} \rightarrow E$ and $I_{i}, J_{i}: E \rightarrow E$ are appropriate mappings satisfying certain conditions to be specified later, where $D_{1}=\{(t, s) \in[0, T] \times[0, T]: s \leq t\}$ and $L(K, E)$ denotes the space of linear bounded operators from $K$ into $E$. For study of differential equations with infinite delay, we refer to the $[4,5]$.

The rest of the paper is organized as follows. Section 2 provides some basic notations and preliminaries. Section 3 establishes the existence of the mild solution to the nonlocal stochastic fractional system involving impulsive effects by utilizing 
stochastic analysis, resolvent operator and fixed point theorem. Section 4 derives a set of sufficient conditions proving approximate controllability approximate of the stochastic system. An example is also considered at the end of the article illustrating the application of obtained results.

\section{PRELIMINARIES}

In this section, some basic definitions, preliminaries, theorems and lemmas and assumptions which will be used to prove existence result, are stated.

Throughout the work, we assume that $\left(E,\|\cdot\|,<\cdot, \cdot>_{E}\right)$ and $(K,\|\cdot\|,<$ $\left.\cdot, \cdot>_{K}\right)$ are separable Hilbert spaces. The symbol $C([0, T] ; E)$ stands for the Banach space of all the continuous functions from $[0, T]$ into $E$ equipped with the norm $\|z(t)\|_{C}=\sup _{t \in[0, T]}\|z(t)\|_{E}$ and $L^{p}((0, T) ; E)$ stands for Banach space of all Bochner-measurable functions from $(0, T)$ to $E$ with the norm

$$
\|z\|_{L^{p}}=\left(\int_{(0, T)}\|z(s)\|_{E}^{p} d s\right)^{1 / p} .
$$

Let $(\Omega, \mathcal{F}, \mathbb{P})$ be a complete probability space with a normal filtration $\left\{\mathcal{F}_{t}\right\}_{t \geq 0}$ that satisfies the right continuity and $\mathcal{F}_{0}$ containing all $\mathbb{P}$-null sets of $\mathcal{F}$. An $E$-valued random variable is an $\mathcal{F}$-measurable function $u(t): \Omega \rightarrow E$ and the collection of random variables $\mathcal{U}=\left\{u(t, w):\left.\Omega \rightarrow E\right|_{t \in[0, T]}\right\}$ is called a stochastic process. In general, we can write $u(t)$ instead of $u(t, w)$ and $u(t):[0, T] \rightarrow E$ in the space of $\mathcal{U}$. Assume that $\{w(t)\}_{t \geq 0}$ is a $K$-valued Wiener process with finite trace nuclear covariance operator $\mathcal{Q}$ and $\operatorname{Tr}(\mathcal{Q})=\sum_{i=1}^{\infty} \lambda_{i}<\infty$ that fulfills $\mathcal{Q} e_{i}=\lambda_{i} e_{i}$, where $\left\{e_{i}\right\}_{i=1}^{\infty}$ is a complete orthonormal basis of $K$. Thus, $w(t)=\sum_{i=1}^{\infty} \sqrt{\lambda_{i}} \beta_{i}(t) e_{i}$. Here $\left\{\beta_{i}(t)\right\}_{i=1}^{\infty}$ are mutually independent one-dimensional standard Wiener processes. Suppose that $\mathcal{F}_{t}=\sigma\{w(s): 0 \leq s \leq t\}$ is the $\sigma$-algebra generated by $W$ and $\mathcal{F}_{t}=\mathcal{F}$. Also, we define

$$
\|\phi\|_{\mathcal{Q}}^{2}=\operatorname{Tr}\left(\phi \mathcal{Q} \phi^{*}\right)=\sum_{i=1}^{\infty}\left\|\sqrt{\lambda_{i}} \phi e_{i}\right\|^{2}, \quad \text { for } \phi \in L(K, E) .
$$

If $\|\phi\|_{\mathcal{Q}}<\infty$, then $\phi$ is said to be a $\mathcal{Q}$ Hilbert-Schmidt operator. The space $L_{\mathcal{Q}}(K, E)=L_{2}^{0}=L_{2}(K, E)$ represents the space of all $\mathcal{Q}$-Hilbert-Schmidt operators $\phi: K \rightarrow E$. The notation $L_{2}(\Omega, \mathcal{F}, \mathbb{P}, E)=L_{2}(\Omega, E)$ stands for the Banach space of all strongly measurable, square integrable $\mathrm{H}$-valued random variables with the norm $\|y(\cdot)\|_{L_{2}}=\left(\mathbb{E}\|y(\cdot, w)\|_{E}^{2}\right)^{1 / 2}$, where the $\mathbb{E}$ is known as expectation defined by $\mathbb{E}(y)=\int_{\Omega} y(w) d \mathbb{P}$.

Let $\bar{J}=(-\infty, T]$. The notation $C\left(\bar{J}, L_{2}(\Omega, E)\right)$ stands for the Banach space of all continuous maps from $\bar{J}$ into $L_{2}(\Omega, E)$ fulfilling the condition $\sup _{t \in \bar{J}} \mathbb{E}\|y(t)\|^{2}<\infty$. 
To treat the impulsive neutral stochastic fractional differential equation, we present the abstract space phase $\mathfrak{B}_{v}$. Let $v:(-\infty, 0] \rightarrow(0, \infty)$ be assumed to be a continuous function with $l=\int_{-\infty}^{0} v(t) d t<\infty$. For any $c>0$, we define

$\mathfrak{B}_{v}=\left\{\varphi:(-\infty, 0] \rightarrow E\right.$ such that $\left(\mathbb{E}|\varphi(\zeta)|^{2}\right)^{1 / 2}$ is a bounded and measurable on

$$
\left.[-c, 0] \text { and } \int_{-\infty}^{0} v(s) \sup _{\zeta \in[0, s]}\left(\mathbb{E}|\varphi(\zeta)|^{2}\right)^{1 / 2} d s<\infty\right\} \text {. }
$$

It is not difficult to verify that $\mathfrak{B}_{v}$ is a Banach space endowed with the norm

$$
\|\varphi\|_{\mathfrak{B}_{v}}=\int_{-\infty}^{0} v(s) \sup _{s \leq \zeta \leq 0}\left(\mathbb{E}|\varphi(\zeta)|^{2}\right)^{1 / 2} d s, \text { for all } \varphi \in \mathfrak{B}_{v}
$$

i.e., $\left(\mathfrak{B}_{v},\|\cdot\|_{\mathfrak{B}_{v}}\right)$ is a Banach space [5].

Next, we consider the space

$$
\begin{array}{r}
\mathfrak{B}_{T}=\left\{u:(-\infty, T] \rightarrow E \text { such that }\left.u\right|_{J_{k}} \in C\left(J_{k}, E\right) \text { and there exist } u\left(t_{k}^{-}\right)=u\left(t_{k}\right)\right. \\
\text { and } \left.u\left(t_{k}^{+}\right), u_{0}=\phi \in \mathfrak{B}_{v}, k=0,1, \cdots, m\right\} .
\end{array}
$$

Here $\left.u\right|_{J_{k}}$ denotes the restriction of $u$ to $J_{k}=\left(t_{k}, t_{k+1}\right], k=1, \cdots, m$ and the notation $C\left(J_{k}, E\right)$ stands for the space of all continuous $E$-valued stochastic processes $\{u(t)$ : $\left.t \in J_{k}, k=1, \cdots, m\right\}$. Let $\|\cdot\|_{T}$ be a seminorm in $\mathfrak{B}_{T}$ which is defined by

$$
\|u\|_{T}=\left\|u_{0}\right\|_{\mathfrak{B}_{v}}+\sup _{s \in[0, T]}\left(\mathbb{E}\left(|u(s)|^{2}\right)\right)^{1 / 2}, u \in \mathfrak{B}_{T} .
$$

Now, we give the following lemma [29].

Lemma 2.1. [28]If $u \in \mathfrak{B}_{T}$, then for $t \in J, u_{t} \in \mathfrak{B}_{v}$. Moreover,

$$
l\left(E\|u(t)\|^{2}\right)^{1 / 2} \leq\left\|u_{t}\right\|_{\mathfrak{B}_{v}} \leq l \sup _{s \in[0, t]}\left(E\|u(s)\|^{2}\right)^{1 / 2}+\left\|u_{0}\right\|_{\mathfrak{B}_{v}}
$$

here $l=\int_{-\infty}^{0} v(s) d s<\infty$.

Now, we state some basic definitions and properties of fractional calculus.

Definition 2.2. The Riemann-Liouville fractional integral operator $J$ of order $q>0$ is defined as

$$
J_{t}^{q} F(t)=\frac{1}{\Gamma(q)} \int_{0}^{t}(t-s)^{q-1} F(s) d s,
$$

where $F \in L^{1}((0, T), E)$.

Definition 2.3. The Riemann-Liouville fractional derivative is given as

$$
D_{t}^{q} F(t)=D_{t}^{m} J_{t}^{m-q} F(t), m-1<q<m, m \in \mathbb{N},
$$


where $D_{t}^{m}=\frac{d^{m}}{d t^{m}}, F \in L^{1}((0, T) ; E), J_{t}^{m-q} \in W^{m, 1}((0, T) ; E)$. Here the notation $W^{m, 1}((0, T) ; E)$ stands for the Sobolev space defined as

$$
\begin{aligned}
W^{m, 1}((0, T) ; E)= & \left\{y \in E: \exists z \in L^{1}((0, T) ; E): y(t)=\sum_{k=0}^{m-1} d_{k} \frac{t^{k}}{k !}\right. \\
& \left.+\frac{t^{m-1}}{(m-1) !} * z(t), t \in(0, T)\right\} .
\end{aligned}
$$

Note that $z(t)=y^{m}(t), d_{k}=y^{k}(0)$.

Definition 2.4. The Caputo fractional derivative is given as

$$
{ }^{C} D_{t}^{\alpha} F(t)=\frac{1}{\Gamma(m-\alpha)} \int_{0}^{t}(t-s)^{m-\alpha-1} F^{m}(t) d t, \quad m-1<\alpha<m .
$$

where $F \in C^{m-1}((0, T), E) \cap L^{1}((0, T), E)$.

Definition 2.5. The definition of one parameter Mittag-Leffler function is given by

$$
E_{\alpha}(z)=\sum_{k=0}^{\infty} \frac{z^{k}}{\Gamma(\alpha k+1)}
$$

and two parameter function of Mittag-Leffler type is defined by

$$
E_{\alpha, \beta}(z)=\sum_{k=0}^{\infty} \frac{z^{k}}{\Gamma(\alpha k+\beta)}=\frac{1}{2 \pi i} \int_{C} \frac{\mu^{\alpha-\beta} e^{\mu}}{\mu^{a}-z} d \mu, \quad 0<\alpha, \beta, z \in \mathbb{C},
$$

where $C$ is a contour which starts and ends at $-\infty$ and encircles the disc $|\mu| \leq|z|^{1 / 2}$ counter clockwise. The Laplace transform of the Mittag-Leffler is defined as

$$
L\left(t^{\beta-1} E_{\alpha, \beta}\left(-\rho^{\alpha} t^{\alpha}\right)\right)=\frac{\lambda^{\alpha-\beta}}{\lambda^{\alpha}+\rho^{\alpha}}, \quad \operatorname{Re} \lambda>\rho^{1 / \alpha}, \rho>0 .
$$

For more details, we refer to [3].

Definition 2.6. [37]Let $A: D(A) \subset E \rightarrow E$ be a closed linear operator. $A$ is said to be sectorial operator of type $(M, \theta, \mu)$ if there exist $0<\theta<\pi / 2, M>0$ and $\mu \in \mathbb{R}$ such that the $q$-resolvent of $A$ exists outside the sector

$$
\mu+S_{\theta}=\{\mu+\lambda: \lambda \in \mathbb{C},|\arg (-\lambda)|<\theta\},
$$

and

$$
\left\|(\lambda I-A)^{-1}\right\| \leq \frac{M}{|\lambda-\mu|}, \quad \lambda \notin \mu+S_{\theta} .
$$

Definition 2.7. [37]Let $A$ be a densely defined operator in $E$ that satisfies the following conditions: 
(i) For some $0<\theta<\pi / 2, \mu+S_{\theta}=\{\mu+\lambda: \lambda \in \mathbb{C},|\operatorname{Arg}(-\lambda)|<\theta\}$,

(ii) There is a constant $M>0$ such that

$$
\left\|(\lambda I-A)^{-1}\right\| \leq \frac{M}{|\lambda-\mu|}, \quad \lambda \notin \mu+S_{\theta} .
$$

Then, $A$ is the infinitesimal generator of a semigroup $\mathcal{T}(t)$ fulfilling $\|\mathcal{T}(t)\| \leq C$. Moreover,

$$
\mathcal{T}(t)=\frac{1}{2 \pi i} \int_{\widetilde{\Gamma}} e^{\lambda t} R(\lambda, A) d \lambda
$$

where $\widetilde{\Gamma}$ is a suitable path for $\lambda \notin \mu+S_{\theta}$ and $\lambda \in \widetilde{\Gamma}$.

Definition 2.8. [37]A closed linear operator $A: D(A) \subset E \rightarrow E$ said to be a sectorial operator of type $(M, \theta, q, \mu)$ if there exist $0<\theta<\pi / 2, M>0$ and $\mu \in \mathbb{R}$ such that the $q$-resolvent of A exists outside the sector

$$
\mu+S_{\theta}=\left\{\mu+\lambda^{q}: \lambda \in \mathbb{C},\left|\operatorname{Arg}\left(-\lambda^{q}\right)\right|<\theta\right\}
$$

and

$$
\left\|\left(\lambda^{q} I-A\right)^{-1}\right\| \leq M /\left|\lambda^{q}-\mu\right|, \quad \lambda^{q} \notin \mu+S_{\theta} .
$$

Remark 2.9. If $A$ is a sectorial operator of type $(M, \theta, q, \mu)$, then it is not difficult to see that $A$ is the infinitesimal generator of a $q$-resolvent family $\left\{S_{q}(t)\right\}_{t \geq 0}$ in a Banach space and

$$
\begin{aligned}
S_{q}(t) & =\frac{1}{2 \pi i} \int_{\widetilde{\Gamma}} e^{\lambda t} \lambda^{q-1} R\left(\lambda^{q}, A\right) d \lambda, \\
K_{q}(t) & =\frac{1}{2 \pi i} \int_{\widetilde{\Gamma}} e^{\lambda t} \lambda^{q-2} R\left(\lambda^{q}, A\right) d \lambda, \\
R_{q}(t) & =\frac{1}{2 \pi i} \int_{\widetilde{\Gamma}} e^{\lambda t} R\left(\lambda^{q}, A\right) d \lambda,
\end{aligned}
$$

and $\widetilde{\Gamma}$ is a suitable path.

Now, the definition of the mild solution to equation (1.1) is presented.

Definition 2.10. A stochastic process $u(t):(-\infty, T] \rightarrow E$ is said to be mild solution of equation (1.1) if:

(i) $u(t)$ is measurable and $\mathcal{F}_{t}$ adapted for all $t \in(-\infty, T]$ having càdlàg path on $t \geq 0$ almost surely.

(ii) $u(t)$ is $\mathfrak{B}_{v}$ valued and the restriction of $u(\cdot)$ to the interval $\left(t_{i}, t_{i+1}\right], i=$ $1, \cdots, m$ is continuous. 
(iii) For each $t \geq 0, u(t)$ satisfies the following integral equation

$$
u(t)=\left\{\begin{array}{l}
\phi(t), \quad t \in(-\infty, 0], \\
S_{q}(t)[\phi(0)-g(u)]+K_{q}(t)\left[u_{1}-h(u)\right] \\
-\int_{0}^{t} K_{q}(t-s) G\left(s, u_{s}, \int_{0}^{s} a_{1}\left(s, \tau, u_{\tau}\right) d \tau\right) d s \\
+\int_{0}^{t} R_{q}(t-s) B x(s) d s+\int_{0}^{t} R_{q}(t-s) F\left(s, u_{s}, \int_{0}^{s} a_{2}\left(s, \tau, u_{\tau}\right) d \tau\right) d s \\
+\int_{0}^{t} R_{q}(t-s) H\left(s, u_{s}, \int_{0}^{s} a_{3}\left(s, \tau, u_{\tau}\right) d \tau\right) d W(s) \\
+\sum_{0<t_{i}<t} S_{q}\left(t-t_{i}\right) I_{i}\left(u\left(t_{i}\right)\right) \\
+\sum_{0<t_{i}<t} K_{q}\left(t-t_{i}\right) J_{i}\left(u\left(t_{i}\right)\right), \quad t \in[0, T] .
\end{array}\right.
$$

Lemma 2.11. For any $\bar{u}_{T} \in L^{2}\left(\mathcal{F}_{T}, E\right)$, there exists $\sigma(\cdot) \in L_{\mathcal{F}}^{2}\left(\Omega, L^{2}\left([0, T], L_{2}^{0}\right)\right)$ such that $\bar{u}_{T}=\mathbb{E} u_{T}+\int_{0}^{T} \sigma(s) d W(s)$.

Define the operator $\Gamma_{0}^{T}: E \rightarrow E$ associated with the linear system of (1.1) as

$$
\Gamma_{0}^{T}=\int_{0}^{T} R_{q}(T-s) B B^{*} R_{q}^{*}(T-s) d s, \quad R\left(\lambda, \Gamma_{0}^{T}\right)=\left(\lambda I+\Gamma_{0}^{T}\right)^{-1},
$$

It is convenient at this point to define operators

$$
\begin{aligned}
\Gamma_{\tau}^{T} & =\int_{\tau}^{T} R_{q}(T-s) B B^{*} R_{q}^{*}(T-s) d s, \\
\Gamma_{t_{k-1}}^{t_{k}} & =\int_{t_{k-1}}^{t_{k}} R_{q}\left(t_{k}-s\right) B B^{*} R_{q}^{*}\left(t_{k}-s\right) d s, \\
R\left(\lambda, \Gamma_{t_{k-1}}^{t_{k}}\right) & =\left(\lambda I+\Gamma_{t_{k-1}}^{t_{k}}\right)^{-1} \text { for } a>0, \quad k=1, \cdots, m
\end{aligned}
$$

where $B^{*}$ denotes the adjoint of $B,\|B\|=M_{B}$ and $R_{q}^{*}(t)$ is the self adjoint of $R_{q}(t)$. Generally, we consider $x(t)=x^{\lambda}(t, u)=B^{*} R_{q}^{*}(T-t) R\left(\lambda, \Gamma_{0}^{T}\right) k(u(\cdot))$, where

$$
k(u(\cdot))=\left\{\begin{array}{l}
\mathbb{E} u_{T}+\int_{0}^{T} \sigma(s) d W(s)-S_{q}(T)[\phi(0)-g(u)]-K_{q}(T)\left[u_{1}-h(u)\right] \\
+\int_{0}^{T} K_{q}(T-s) G\left(s, u_{s}, \int_{0}^{s} a_{1}\left(s, \tau, u_{\tau}\right) d \tau\right) d s \\
-\int_{0}^{T} R_{q}(T-s) F\left(s, u_{s}, \int_{0}^{s} a_{2}\left(s, \tau, u_{\tau}\right) d \tau\right) d s \\
-\int_{0}^{T} R_{q}(T-s) H\left(s, u_{s}, \int_{0}^{s} a_{3}\left(s, \tau, u_{\tau}\right) d \tau\right) d s, \quad t \in\left[0, t_{1}\right] \\
\mathbb{E} u_{T}+\int_{0}^{T} \sigma(s) d W(s)-S_{q}(T)[\phi(0)-g(u)]-K_{q}(T)\left[u_{1}-h(u)\right] \\
+\int_{0}^{T} K_{q}(T-s) G\left(s, u_{s}, \int_{0}^{s} a_{1}\left(s, \tau, u_{\tau}\right) d \tau\right) d s \\
-\int_{0}^{T} R_{q}(T-s) F\left(s, u_{s}, \int_{0}^{s} a_{2}\left(s, \tau, u_{\tau}\right) d \tau\right) d s \\
-\int_{0}^{T} R_{q}(T-s) H\left(s, u_{s}, \int_{0}^{s} a_{3}\left(s, \tau, u_{\tau}\right) d \tau\right) d W(s) \\
-\sum_{i=1}^{m} S_{q}\left(T-t_{i}\right) I_{i}\left(u\left(t_{i}\right)\right) \\
-\sum_{i=1}^{m} K_{q}\left(T-t_{i}\right) J_{i}\left(u\left(t_{i}\right)\right), t \in\left(t_{i}, t_{i+1}\right], i=1, \cdots, m .
\end{array}\right.
$$


Let $u(t, \phi, x)$ be the state value of system (1.1) at time $t$ corresponding to the control $x \in L_{2}^{\mathcal{F}}(J, X)$. In particular, the state of system (1.1) at $t=T, u(T, \phi, x)$ is known as the terminal state with control $x$. The set $\mathfrak{R}(T, \phi, x)=\{u(T, \phi, x): x \in$ $\left.L_{2}^{\mathcal{F}}([0, T], X)\right\}$ is said to be reachable set of system (1.1).

Definition 2.12. The system (1.1) is said to be approximately controllable on $[0, T]$ if $\overline{\mathfrak{R}(T, \phi, x)}=L_{2}(\Omega, \mathcal{F}, E)$, where $\overline{\mathfrak{R}(T, \phi, x)}$ denotes the closure of the reachable set.

Now, we state the Krasnoselskii-Schaefer fixed point theorem which is our main tool to establish our existence result.

Theorem 2.13. [26] Let $\Psi_{1}$ and $\Psi_{2}$ be two operators defined on $E$ such that

(i) $\Psi_{1}$ is contraction,

(ii) $\Psi_{2}$ is completely continuous,

then, either

(1) the operator equation $\Psi_{1} y+\Psi_{2} y=y$ has a solution, or

(2) the set $\mathcal{G}=\left\{y \in E: \lambda_{1} \Psi_{1}\left(y / \lambda_{1}\right)+\lambda_{1} \Psi_{2} y=y\right\}$ is unbounded for $\lambda_{1} \in(0,1)$.

\section{EXISTENCE OF MILD SOLUTIONS}

For proving existence of the mild solution, we need to impose following assumptions on the data of the system (1.1)-(1.3).

(A1) The operator $S_{q}(t), K_{q}(t)$ and $R_{q}(t), t \geq 0$ generated by $A$ are compact in $\overline{D(A)}$ such that $\sup _{t \in[0, T]}\left\|S_{q}(t)\right\| \leq M, \sup _{t \in[0, T]}\left\|K_{q}(t)\right\| \leq M$ and $\sup _{t \in[0, T]}\left\|R_{q}(t)\right\|$ $\leq M$.

(A2) (i) $G:[0, T] \times \mathfrak{B}_{v} \times E \rightarrow E$ is continuous function and there exists a constant $L_{G}>0$ such that

$$
\mathbb{E}\left\|G\left(t, u_{1}, v_{1}\right)-G\left(t, u_{2}, v_{2}\right)\right\|^{2} \leq L_{G}\left[\left\|u_{1}-u_{2}\right\|_{\mathfrak{B}_{v}}^{2}+\mathbb{E}\left\|v_{1}-v_{2}\right\|^{2}\right],
$$

for all $u_{j}(j=1,2) \in \mathfrak{B}_{v}, v_{j}(j=1,2) \in E$ and $t \in[0, T]$ with

$$
\mathcal{C}_{1}=\sup _{t \in[0, T]}\|G(t, 0,0)\|^{2} .
$$

(ii) There exists a constant $L_{a_{1}}>0$ such that

$\mathbb{E}\left\|\int_{0}^{t}\left[a_{1}\left(t, s, u_{1}\right)-g\left(t, s, u_{2}\right)\right] d s\right\|^{2} \leq L_{a_{1}}\left\|u_{1}-v_{1}\right\|_{\mathfrak{B}_{v}}^{2}, \forall t \in[0, T], u_{1}, v_{1} \in \mathfrak{B}_{v}$, and $\mathcal{C}_{2}=T \sup _{(t, s) \in D_{1}}\left\|a_{1}(t, s, 0)\right\|$. 
(A3) (1) The function $F:[0, T] \times \mathfrak{B}_{v} \times E \rightarrow E$ is a nonlinear function that satisfies following conditions

(i) $t \rightarrow F\left(t, u_{1}, u_{2}\right)$ is measurable for each $\left(u_{1}, u_{2}\right) \in \mathfrak{B}_{v} \times E$.

(ii) $\left(u_{1}, u_{2}\right) \rightarrow F\left(t, u_{1}, u_{2}\right)$ is continuous for almost all $t \in[0, T]$.

(iii) There exist a continuous function $m_{F}:[0, \infty) \rightarrow(0, \infty)$ and a continuous increasing function $\Theta_{F}:[0, \infty) \rightarrow[0, \infty)$ such that

$$
\mathbb{E}\left\|F\left(t, u_{1}, u_{2}\right)\right\|_{E}^{2} \leq m_{F}(t) \Theta_{F}\left(\left\|u_{1}\right\|_{\mathfrak{B}_{v}}^{2}+\mathbb{E}\left\|u_{2}\right\|_{E}^{2}\right),
$$

for all $\left(u_{1}, u_{2}\right) \in \mathfrak{B}_{v} \times E$ and $t \in[0, T]$.

(2) For each $(t, s) \in D_{1}$, the function $a_{2}(t, s, \cdot): \mathfrak{B}_{v} \rightarrow E$ is continuous and $a_{2}(\cdot, \cdot, u): D_{1} \rightarrow E$ is measurable for each $u \in \mathfrak{B}_{v}$. There exist a constant $L_{a_{2}}>0$ and a continuous increasing function $\mathcal{W}_{a_{2}}:[0, \infty) \rightarrow[0, \infty)$ such that

$$
\mathbb{E}\left\|a_{2}(t, s, u)\right\|^{2} \leq L_{a_{2}} \mathcal{W}_{a_{2}}\left(\|u\|_{\mathfrak{B}_{v}}^{2}\right), \quad \forall u \in \mathfrak{B}_{v}
$$

(A4) (1). The function $H:[0, T] \times \mathfrak{B}_{v} \times E \rightarrow \mathcal{L}(K, E)$ satisfies the Carathéodory condition and there exist a function $m_{H}(t) \in L_{l o c}\left(J, \mathbb{R}^{+}\right)$and a nondecreasing function $\Theta_{H}:[0, \infty) \rightarrow(0, \infty)$ such that

$$
\begin{aligned}
\mathbb{E}\left\|H\left(t, u_{1}, u_{2}\right)\right\|_{E}^{2} \leq m_{H}(t) \Theta_{H}\left(\left\|u_{1}\right\|_{\mathfrak{B}_{v}}^{2}+\mathbb{E}\left\|u_{2}\right\|_{E}^{2}\right), & \\
& \forall\left(u_{1}, u_{2}\right) \in \mathfrak{B}_{v} \times E, \quad t \in[0, T] .
\end{aligned}
$$

(2). For each $(t, s) \in D_{1}$, the function $a_{3}(t, s, \cdot): \mathfrak{B}_{v} \rightarrow E$ is continuous and the map $a_{3}(\cdot, \cdot, z): D_{1} \rightarrow E$ measurable for each $z \in \mathfrak{B}_{v}$. There is a constant $m_{a_{3}}>0$ such that $\mathbb{E}\left\|a_{3}(t, s, z)\right\|^{2} \leq m_{a_{3}} \Theta_{a_{3}}\left(\|z\|_{\mathfrak{B}_{v}}^{2}\right)$, for all $(t, s) \in D_{1}$ and $z \in \mathfrak{B}_{v}$, where $\mathcal{W}_{a_{3}}:[0, \infty) \rightarrow[0, \infty)$ is a nondecreasing function.

(A5) The functions $I_{i}, J_{i}: E \rightarrow E(i=1, \cdots, m)$ are completely continuous functions and there are positive constant $\Phi_{1}^{i}, \Psi_{2}^{i}>0$ such that

$$
\mathbb{E}\left\|I_{i}(z)\right\|_{E}^{2} \leq \Phi_{1}^{i}, \quad \mathbb{E}\left\|J_{i}(z)\right\|_{E}^{2} \leq \Psi_{2}^{i}, \quad \forall z \in E .
$$

(A6) The function $g, h: \mathfrak{B}_{v} \rightarrow E$ are continuous and there exist some constant $\widehat{L}_{g}, \widehat{L}_{h}>0$ and $\widehat{L}_{g}^{1}, \widehat{L}_{h}^{1}>0$ such that

$$
\begin{aligned}
\mathbb{E}\left\|g\left(z_{1}\right)-g\left(z_{2}\right)\right\|_{E}^{2} & \leq \widehat{L}_{g}\left\|z_{1}-z_{2}\right\|_{\mathfrak{B}_{v}}^{2} \\
\mathbb{E}\|g(z)\| & \leq \widehat{L}_{g}\|z\|_{\mathfrak{B}_{v}}^{2}+\widehat{L}_{g}^{1}, \\
\mathbb{E}\left\|h\left(z_{1}\right)-h\left(z_{2}\right)\right\|_{E}^{2} & \leq \widehat{L}_{h}\left\|z_{1}-z_{2}\right\|_{\mathfrak{B}_{v}}^{2} \\
\mathbb{E}\|h(z)\| & \leq \widehat{L}_{h}\|z\|_{\mathfrak{B}_{v}}^{2}+\widehat{L}_{h}^{1}
\end{aligned}
$$

for all $z_{1}, z_{2}, z \in \mathfrak{B}_{v}$. 
$(A 7)$

$$
\begin{gathered}
\int_{0}^{T} \widehat{m}(s) d s \leq \int_{\xi(0)}^{\infty} \frac{d s}{\Theta_{F}(s)+\Theta_{H}(s)+\Theta(s)}, \text { where } \xi(0)=\frac{\widetilde{M}}{1-\widehat{N}}, \widehat{m}(t)=\max \{\bar{m}(t), T \mathfrak{L}\} \\
\bar{m}(t)=\max \left\{\frac{32}{(1-\widetilde{N})} \times l^{2} M^{2} T^{2} \times m_{F}(t), \frac{32}{(1-\widetilde{N})} l^{2} M^{2} \operatorname{Tr}(Q) T \times m_{H}(t)\right\} \\
\mathfrak{L}=\max \left\{L_{a_{2}}, m_{a_{3}}\right\}, \quad \Theta(y)=\max \left\{\mathcal{W}_{a_{2}}(y), \Theta_{a_{3}}(y)\right\},
\end{gathered}
$$

and $\widetilde{M}, \widetilde{N}$ are defined later.

Theorem 3.1. The system (1.1) has a mild solution on $(-\infty, T]$ if the assumptions (A1)-(A7) are satisfied and

$$
\Theta=3 M^{2}\left[L_{g}+L_{h}+T^{2} L_{G}\left(1+L_{g}\right)\right]<1
$$

Proof. We first consider the operator $\Upsilon: \mathfrak{B}_{T} \rightarrow \mathfrak{B}_{T}$ defined by

$$
\Upsilon u(t)=\left\{\begin{array}{l}
\phi(t), \quad t \in(-\infty, 0] \\
S_{q}(t)[\phi(0)-g(u)]+K_{q}(t)\left[u_{1}-h(u)\right] \\
-\int_{0}^{t} K_{q}(t-s) G\left(s, u_{s}, \int_{0}^{s} a_{1}\left(s, \tau, u_{\tau}\right) d \tau\right) d s \\
+\int_{0}^{t} R_{q}(t-s) B x(s) d s+\int_{0}^{t} R_{q}(t-s) F\left(s, u_{s}, \int_{0}^{s} a_{2}\left(s, \tau, u_{\tau}\right) d \tau\right) d s \\
+\int_{0}^{t} R_{q}(t-s) H\left(s, u_{s}, \int_{0}^{s} a_{3}\left(s, \tau, u_{\tau}\right) d \tau\right) d W(s) \\
+\sum_{0<t_{i}<t} S_{q}\left(t-t_{i}\right) I_{i}\left(u\left(t_{i}\right)\right) \\
+\sum_{0<t_{i}<t} K_{q}\left(t-t_{i}\right) J_{i}\left(u\left(t_{i}\right)\right), \quad t \in[0, T]
\end{array}\right.
$$

We shall show that the operator $\Upsilon$ has a fixed point in the space $\mathfrak{B}_{T}$ which is the mild solution of (1.1).

For $\phi \in \mathfrak{B}_{v}$, we define $\widehat{\phi}$ by

$$
y(t)=\left\{\begin{array}{l}
\phi(t), \quad t \in(-\infty, 0] \\
S_{q}(t) \phi(0), \quad t \in[0, T] .
\end{array}\right.
$$

Then $y \in \mathfrak{B}_{T}$. We also define a function

$$
\widehat{z}(t)=\left\{\begin{array}{l}
0, \quad t \in(-\infty, 0], \\
z(t), \quad t \in[0, T],
\end{array}\right.
$$

for every $z \in C(J, E)$. We set $u(t)=y(t)+\widehat{z}(t)$ for each $t \in[0, T]$. It is clear that $u$ is the solution for problem (1.1)-(1.3) if and only if $z$ satisfies $z_{0}=0, t \in(-\infty, 0]$ and

$$
\begin{aligned}
z(t)= & S_{q}(t)[-g(y+\widehat{z})]+K_{q}(t)\left[u_{1}-h(y+\widehat{z})\right] \\
& -\int_{0}^{t} K_{q}(t-s) G\left(s, y_{s}+\widehat{z}_{s}, \int_{0}^{s} a_{1}\left(s, \tau, y_{\tau}+\widehat{z}_{\tau}\right) d \tau\right) d s
\end{aligned}
$$




$$
\begin{aligned}
& +\int_{0}^{t} R_{q}(t-s) B x(s) d s+\int_{0}^{t} R_{q}(t-s) F\left(s, y_{s}+\widehat{z}_{s}, \int_{0}^{s} a_{2}\left(s, \tau, y_{\tau}+\widehat{z}_{\tau}\right) d \tau\right) d s \\
& +\int_{0}^{t} R_{q}(t-s) H\left(s, y_{s}+\widehat{z}_{s}, \int_{0}^{s} a_{3}\left(s, \tau, y_{\tau}+\widehat{z}_{\tau}\right) d \tau\right) d W(s) \\
& +\sum_{0<t_{i}<t} S_{q}\left(t-t_{i}\right) I_{i}\left(y\left(t_{i}\right)+\widehat{z}\left(t_{i}\right)\right)+\sum_{0<t_{i}<t} K_{q}\left(t-t_{i}\right) J_{i}\left(y\left(t_{i}\right)+\widehat{z}\left(t_{i}\right)\right),
\end{aligned}
$$

$t \in[0, T]$

Let $\mathfrak{B}_{T}^{0}=\left\{z \in \mathfrak{B}_{T}: z_{0}=0 \in \mathfrak{B}_{v}\right\}$ and for any $z \in \mathfrak{B}_{T}^{0}$, we get

$$
\|z\|_{T}=\left\|z_{0}\right\|_{\mathfrak{B}_{v}}+\sup _{t \in[0, T]}\left(\mathbb{E}\|z(t)\|^{2}\right)^{1 / 2}=\sup _{t \in[0, T]}\left(\mathbb{E}\|z(t)\|^{2}\right)^{1 / 2}
$$

It can be easy to verify that $\left(\mathfrak{B}_{T}^{0},\|\cdot\|_{\mathfrak{B}_{T}^{0}}\right)$ is a Banach space.

Now, we define the operator $\Psi: \mathfrak{B}_{T}^{0} \rightarrow \mathfrak{B}_{T}^{0}$ by

$$
\Psi z(t)=\left\{\begin{array}{l}
0, \quad t \in(-\infty, 0] \\
S_{q}(t)[-g(y+\widehat{z})]+K_{q}(t)\left[u_{1}-h(y+\widehat{z})\right]-\int_{0}^{t} K_{q}(t-s) \\
\times G\left(s, y_{s}+\widehat{z}_{s}, \int_{0}^{s} a_{1}\left(s, \tau, y_{\tau}+\widehat{z}_{\tau}\right) d \tau\right) d s+\int_{0}^{t} R_{q}(t-s) B x(s) d s \\
+\int_{0}^{t} R_{q}(t-s) F\left(s, y_{s}+\widehat{z}_{s}, \int_{0}^{s} a_{2}\left(s, \tau, y_{\tau}+\widehat{z}_{\tau}\right) d \tau\right) d s \\
+\int_{0}^{t} R_{q}(t-s) H\left(s, y_{s}+\widehat{z}_{s}, \int_{0}^{s} a_{3}\left(s, \tau, y_{\tau}+\widehat{z}_{\tau}\right) d \tau\right) d W(s) \\
+\sum_{0<t_{i}<t} S_{q}\left(t-t_{i}\right) I_{i}\left(y\left(t_{i}\right)+\widehat{z}\left(t_{i}\right)\right) \\
+\sum_{0<t_{i}<t} K_{q}\left(t-t_{i}\right) J_{i}\left(y\left(t_{i}\right)+\widehat{z}\left(t_{i}\right)\right), t \in[0, T]
\end{array}\right.
$$

In order to prove the existence result, it is enough to prove that $\Psi$ has a fixed point. To this end, we introduce the decomposition of operator $\Psi$ as

$$
\begin{aligned}
\Psi_{1} z(t)=S_{q}(t)[-g(y+\widehat{z})]+K_{q}(t)\left[u_{1}-h(y+\widehat{z})\right] & \\
& -\int_{0}^{t} K_{q}(t-s) G\left(s, y_{s}+\widehat{z}_{s}, \int_{0}^{s} a_{1}\left(s, \tau, y_{\tau}+\widehat{z}_{\tau}\right) d \tau\right) d s
\end{aligned}
$$

for $t \in[0, T]$, and

$$
\begin{aligned}
\Psi_{2} z(t)= & \int_{0}^{t} R_{q}(t-s) B x(s) d s+\int_{0}^{t} R_{q}(t-s) F\left(s, y_{s}+\widehat{z}_{s}, \int_{0}^{s} a_{2}\left(s, \tau, y_{\tau}+\widehat{z}_{\tau}\right) d \tau\right) d s \\
& +\int_{0}^{t} R_{q}(t-s) H\left(s, y_{s}+\widehat{z}_{s}, \int_{0}^{s} a_{3}\left(s, \tau, y_{\tau}+\widehat{z}_{\tau}\right) d \tau\right) d W(s) \\
& +\sum_{0<t_{i}<t} S_{q}\left(t-t_{i}\right) I_{i}\left(y\left(t_{i}\right)+\widehat{z}\left(t_{i}\right)\right) \\
& +\sum_{0<t_{i}<t} K_{q}\left(t-t_{i}\right) J_{i}\left(y\left(t_{i}\right)+\widehat{z}\left(t_{i}\right)\right), \quad t \in[0, T] .
\end{aligned}
$$


Set $\mathcal{B}_{r}=\left\{y \in \mathfrak{B}_{T}^{0}: \mathbb{E}\|y\|_{\mathfrak{B}_{T}^{0}}^{2} \leq r, r>0\right\}$. Clearly, $\mathcal{B}_{r}$ a bounded closed convex set in $\mathfrak{B}_{T}^{0}$. For $y \in \mathcal{B}_{r}$ and Lemma 2.1, we have that

$$
\begin{aligned}
\left\|y_{t}+\widehat{z}_{t}\right\|_{\mathfrak{B}_{h}}^{2} & \leq 2\left(\left\|y_{t}\right\|_{\mathfrak{B}_{v}}^{2}+\left\|\widehat{z}_{t}\right\|_{\mathfrak{B}_{v}}^{2}\right), \\
& \leq 4\left(l^{2} \sup _{\tau \in[0, t]} \mathbb{E}\|y(\tau)\|_{E}^{2}+\left\|y_{0}\right\|_{\mathfrak{B}_{v}}^{2}\right)+4\left(l^{2} \sup _{\tau \in[0, t]} \mathbb{E}\|\widehat{z}(\tau)\|_{E}^{2}+\left\|\widehat{z}_{0}\right\|_{\mathfrak{B}_{v}}^{2}\right) \\
& \leq 4\left(\|\phi\|_{\mathfrak{B}_{v}}^{2}+l^{2}\left(r+M_{\mathcal{S}}^{2} \mathbb{E}\|\phi(0)\|_{E}^{2}\right)\right) .
\end{aligned}
$$

For establishing the existence result with the help of Theorem 2.13, we show that $\Psi_{1}$ is a contraction while $\Psi_{2}$ is compact operator. To this end, we divide the proof into a several steps.

Step $1 \Phi_{1}$ is a contraction on $\mathfrak{B}_{T}^{0}$.

Let $z_{1}, z_{2} \in \mathfrak{B}_{T}^{0}$ and $t \in\left[0, t_{1}\right]$. Thus, we have

$$
\begin{aligned}
& \mathbb{E}\left\|\left(\Psi_{1} z_{1}\right)(t)-\left(\Psi_{1} z_{2}\right)(t)\right\|_{E}^{2} \leq 3 \mathbb{E}\left\|S_{q}(t)\left[g\left(y+\widehat{z}_{1}\right)-g\left(y+\widehat{z}_{2}\right)\right]\right\|^{2} \\
& +3 \mathbb{E}\left\|K_{q}(t)\left[h\left(y+\widehat{z}_{1}\right)-h\left(y+\widehat{z_{2}}\right)\right]\right\|^{2} \\
& +3 \mathbb{E} \| \int_{0}^{t} K_{q}(t-s)\left[G\left(s, y_{s}+\widehat{z_{1} s}, \int_{0}^{s} a_{1}\left(s, \zeta, y_{\zeta}+\widehat{z_{1 \zeta}}\right) d \zeta\right)\right. \\
& \left.-G\left(s, y_{s}+\widehat{z_{2} s}, \int_{0}^{s} a_{1}\left(s, \zeta, y_{\zeta}+\widehat{z_{2} \zeta}\right) d \zeta\right)\right] d s \|^{2}, \\
\leq & 3 M^{2} L_{g}\left\|\widehat{z_{1}}-\widehat{z_{2}}\right\|^{2}+3 M^{2} L_{h}\left\|\widehat{z_{1}}-\widehat{z_{2}}\right\|^{2}+3 M^{2} T \\
& \times \int_{0}^{t} \mathbb{E} \| G\left(s, y_{s}+\widehat{z_{1} s}, \int_{0}^{s} a_{1}\left(s, \zeta, y_{\zeta}+\widehat{z_{1}}\right) d \zeta\right)-G\left(s, y_{s}+\widehat{z_{2}},\right. \\
& \left.\int_{0}^{s} a_{1}\left(s, \zeta, y_{\zeta}+\widehat{z_{2} \zeta}\right) d \zeta\right) \|^{2} d s, \\
\leq & 3 M^{2} L_{g}\left\|\widehat{z_{1}}-\widehat{z_{2}}\right\|^{2}+3 M^{2} L_{h}\left\|\widehat{z_{1}}-\widehat{z_{2}}\right\|^{2}+3 M^{2} T^{2} L_{G}\left[\left\|\widehat{z_{1 s}}-\widehat{z_{2} s}\right\|_{\mathfrak{B}_{v}}^{2}\right. \\
& \left.+L_{a_{1}}\left\|\widehat{z_{1}}-\widehat{z_{2}}\right\|_{\mathfrak{B}_{v}}^{2}\right], \\
\leq & 3 M^{2}\left(L_{g}+L_{h}\right)\left\|\widehat{z_{1}}-\widehat{z_{2}}\right\|^{2}+3 M^{2} T^{2} L_{G}\left(1+L_{a_{1}}\right) \times\left[\sup _{t \in[0, T]}\left\|\widehat{z_{1}}(t)-\widehat{z_{2}}(t)\right\|^{2}\right. \\
& \left.+\left\|\left(\widehat{z_{1}}\right)_{0}\right\|^{2}+\left\|\left(\widehat{z_{2}}\right)_{0}\right\|^{2}\right], \\
= & 3 M^{2}\left[L_{g}+L_{h}+T^{2} L_{G}\left(1+L_{g}\right)\right]\left\|\widehat{z_{1}}-\widehat{z_{2}}\right\|_{T}^{2} .
\end{aligned}
$$

Using the facts that $\left\|\left(\widehat{z}_{1}\right)_{0}\right\|_{\mathfrak{B}_{v}}^{2}=0$ and $\left\|\left(\widehat{z}_{2}\right)_{0}\right\|_{\mathfrak{B}_{v}}^{2}$. We take the supremum over $t$, we obtain

$$
\left\|\left(\Psi_{1} \widehat{z}_{1}\right)-\left(\Psi_{1} \widehat{z}_{2}\right)\right\|_{T}^{2} \leq \Theta\left\|z_{1}-z_{2}\right\|_{T}^{2},
$$

where $\Theta=3 M^{2}\left[L_{g}+L_{h}+T^{2} L_{G}\left(1+L_{g}\right)\right]$. By inequality (3.2), we conclude that $\Psi_{1}$ is a contraction on $\mathfrak{B}_{T}^{0}$.

Next, we show that $\Psi_{2}$ is completely continuous in following steps. 
Step 2. We first prove that $\Psi_{2}$ maps bounded sets into bounded sets in $\mathfrak{B}_{T}^{0}$. To this end, it is enough to show that there exists a positive constant $\mathbb{M}$ such that for each $z \in \mathcal{B}_{r}$ one has $\mathbb{E}\left\|\left(\Psi_{2} z\right)(t)\right\|_{T}^{2} \leq \mathbb{M}$. Now, for each $z \in \mathcal{B}_{r}$ and for $t \in[0, T]$ $\mathbb{E}\left\|\left(\Psi_{2} z\right)(t)\right\|_{E}^{2}$

$$
\begin{aligned}
& \leq 5 \mathbb{E}\left\|\int_{0}^{t} R_{q}(t-s) B x(s) d s\right\|^{2}+5 \mathbb{E} \| \int_{0}^{t} R_{q}(t-s) F\left(s, y_{s}+\widehat{z}_{s},\right. \\
& \left.\int_{0}^{s} a_{2}\left(s, \tau, y_{\tau}+\widehat{z}_{\tau}\right) d \tau\right) d s\left\|^{2}+5 \mathbb{E}\right\| \int_{0}^{t} R_{q}(t-s) H\left(s, y_{s}+\widehat{z}_{s},\right. \\
& \left.\int_{0}^{s} a_{3}\left(s, \tau, y_{\tau}+\widehat{z}_{\tau}\right) d \tau\right) d W(s)\left\|^{2}+5 \mathbb{E}\right\| \sum_{0<t_{i}<t} S_{q}\left(t-t_{i}\right) I_{i}\left(y\left(t_{i}\right)+\widehat{z}\left(t_{i}\right)\right) \|^{2} \\
& +5 \mathbb{E}\left\|\sum_{0<t_{i}<t} K_{q}\left(t-t_{i}\right) J_{i}\left(y\left(t_{i}\right)+\widehat{z}\left(t_{i}\right)\right)\right\|^{2}, \\
& \leq 5 M_{B}^{2} \int_{0}^{t}\left\|R_{q}(t-s)\right\| d s \int_{0}^{t}\left\|R_{q}(t-s)\right\| \mathbb{E}\|x(s)\| d s+5 \int_{0}^{t}\left\|R_{q}(t-s)\right\| d s \\
& \times \int_{0}^{t}\left\|R_{q}(t-s)\right\| \cdot \mathbb{E}\left\|F\left(s, y_{s}+\widehat{z}_{s}, \int_{0}^{s} a_{2}\left(s, \tau, y_{\tau}+\widehat{z}_{\tau}\right) d \tau\right)\right\|^{2} d s \\
& +5 \int_{0}^{t}\left\|R_{q}(t-s)\right\|^{2} \cdot \mathbb{E}\left\|H\left(s, y_{s}+\widehat{z}_{s}, \int_{0}^{s} a_{3}\left(s, \tau, y_{\tau}+\widehat{z}_{\tau}\right) d \tau\right)\right\|^{2} d s \\
& +5 \sum_{0<t_{i}<t}\left\|S_{q}\left(t-t_{i}\right) I_{i}\left(y\left(t_{i}\right)+\widehat{z}\left(t_{i}\right)\right)\right\|^{2}+5 \sum_{0<t_{i}<t} \mathbb{E}\left\|K_{q}\left(t-t_{i}\right) J_{i}\left(y\left(t_{i}\right)+\widehat{z}\left(t_{i}\right)\right)\right\|^{2}, \\
& \leq 5 M_{B}^{2} M^{2} T^{2} \mathbb{O}+5 M^{2} T^{2} \int_{0}^{t} m_{F}(s) \Theta_{F}\left(r^{*}+T \int_{0}^{s} L_{a_{2}} \mathcal{W}_{a_{2}}\left(r^{*}\right) d \zeta\right) d s \\
& +5 T M^{2} \operatorname{Tr}(\mathcal{Q}) \int_{0}^{t} m_{H}(s) \Theta_{H}\left(r^{*}+T \int_{0}^{s} m_{a_{3}} \Theta_{a_{3}}\left(r^{*}\right) d \zeta\right) d s+5 M^{2} \sum_{i=1}^{m} \Phi_{i}^{1} \\
& +5 M^{2} \sum_{i=1}^{m} \Psi_{i}^{2}=\mathbb{M}
\end{aligned}
$$

where $\mathbb{O}$ is estimated as $\|x(s)\|^{2}$

$$
\begin{aligned}
\leq & \| B^{*} R_{q}^{*}(T-s) R\left(\lambda, \Gamma_{0}^{T}\right)\left\{y_{T}+\widehat{z}_{T}+\int_{0}^{T} \sigma(s) d W(s)-S_{q}(T)[\phi(0)-g(y+\widehat{z})]\right. \\
& -K_{q}(t)\left(u_{1}-h(y+\widehat{z})\right)+\int_{0}^{T} K_{q}(T-s) G\left(s, y_{s}+\widehat{z}_{s}, \int_{0}^{s} a_{1}\left(s, \zeta, y_{\zeta}+\widehat{z}_{t}\right) d \zeta\right) d s \\
& -\sum_{i=1}^{\infty} S_{q}\left(T-t_{i}\right) I_{i}\left(y\left(t_{i}\right)+\widehat{z}\left(t_{i}\right)\right)-\sum_{t_{i}<t} K_{q}\left(s-t_{i}\right) J_{i}\left(y\left(t_{i}\right)+\widehat{z}\left(t_{i}\right)\right) \\
& -\int_{0}^{T} R_{q}(T-s) F\left(s, y_{s}+\widehat{z}_{s}, \int_{0}^{s} a_{2}\left(s, \zeta, y_{\zeta}+\widehat{z}_{\zeta}\right) d \zeta\right) d s-\int_{0}^{T} R_{q}(T-s)
\end{aligned}
$$




$$
\begin{aligned}
& \left.\times H\left(s, y_{s}+\widehat{z}_{s}, \int_{0}^{s} a_{3}\left(s, \zeta, y_{\zeta}+\widehat{z}_{t}\right) d \zeta\right) d W(s)\right\} \|^{2}, \\
\leq & 8 \frac{M_{B}^{2} M^{2}}{\lambda^{2}}\left\{2\left\|y_{T}+\widehat{z}_{T}\right\|^{2}+2 \int_{0}^{T} \mathbb{E}\|\sigma(s)\|_{\mathcal{Q}}^{2} d s+2 M^{2}\left[\|\phi\|_{\mathfrak{B}_{v}}^{2}+\widehat{L}_{g} r^{*}+\widehat{L}_{g}^{1}\right]\right. \\
& +2 M^{2}\left[\left\|u_{1}\right\|^{2}+\widehat{L}_{h} r^{*}+\widehat{L}_{h}^{1}\right]+M^{2} \sum_{i=1}^{m} \Phi_{i}^{1}+M^{2} \sum_{i=1}^{m} \Psi_{i}^{2}+M^{2} T^{2}\left[2 L_{G}\left(1+2 L_{a_{1}}\right) r^{*}\right. \\
& \left.+4 L_{G} \mathcal{C}_{2}+2 \mathcal{C}_{1}\right]+M^{2} T^{2} \int_{0}^{T} m_{F}(s) \Theta_{F}\left(r^{*}+T \int_{0}^{s} L_{a_{2}} \mathcal{W}_{a_{2}}\left(r^{*}\right) d \zeta\right) d s \\
& \left.+M^{2} \operatorname{Tr}(\mathcal{Q}) \times \int_{0}^{T} m_{H}(s) \Theta_{H}\left(r^{*}+T \int_{0}^{s} m_{a_{3}} \Theta_{a_{3}}\left(r^{*}\right) d \zeta\right) d s\right\} \\
& \mathbb{O},
\end{aligned}
$$

where $r^{*}=4\left[\|\phi\|_{\mathfrak{B}_{v}}^{2}+l^{2}\left(r+M_{\mathcal{S}}^{2} \mathbb{E}\|\phi(0)\|_{E}^{2}\right)\right]$.

Thus, we get $\mathbb{E}\left\|\left(\Psi_{2} z\right)(t)\right\|_{E}^{2} \leq \mathbb{M}$.

Step 3. $\Phi_{2}$ is continuous.

Let $\left\{z_{n}\right\}_{n=1}^{\infty}$ be a sequence in $\mathcal{B}_{r}$ with $z_{n} \rightarrow z \in \mathcal{B}_{r}$ as $n \rightarrow \infty$. By the continuity of $F, H, g, h$ and $I_{i}, J_{i}(i=1, \cdots, m)$, we have

$$
\begin{aligned}
F\left(s, y_{s}+\left(\widehat{z}_{n}\right)_{s}, \int_{0}^{s} f\left(s, \tau, y_{\tau}+\left(\widehat{z}_{n}\right)_{\tau}\right) d \tau\right) & \rightarrow F\left(s, y_{s}+\widehat{z}_{s}, \int_{0}^{s} f\left(s, \tau, y_{\tau}+\widehat{z}_{\tau}\right) d \tau\right), \\
H\left(s, y_{s}+\left(\widehat{z}_{n}\right)_{s}, \int_{0}^{s} \varrho\left(s, \tau, y_{\tau}+\left(\widehat{z}_{n}\right)_{\tau}\right) d \tau\right) & \rightarrow H\left(s, y_{s}+\widehat{z}_{s}, \int_{0}^{s} \varrho\left(s, \tau, y_{\tau}+\widehat{z}_{\tau}\right) d \tau\right), \\
g\left(s, y_{s}+\left(\widehat{z}_{n}\right)_{s}, \eta\right) & \rightarrow g\left(s, y_{s}+\widehat{z}_{s}, \eta\right), \\
h\left(s, y_{s}+\left(\widehat{z}_{n}\right)_{s}, \eta\right) & \rightarrow h\left(s, y_{s}+\widehat{z}_{s}, \eta\right), \quad \text { as } n \rightarrow \infty .
\end{aligned}
$$

For $t \in[0, T]$, we get

$\mathbb{E}\left\|\left(\Phi_{2} z_{n}\right)(t)-\left(\Phi_{2} z\right)(t)\right\|_{E}^{2}$

$$
\begin{aligned}
\leq & 5 \mathbb{E}\left\|\sum_{i=1}^{\infty} S_{q}\left(t-t_{i}\right)\left[I_{i}\left(y\left(t_{i}\right)+\widehat{z}_{n}\left(t_{i}\right)\right)-I_{i}\left(y\left(t_{i}\right)+\widehat{z}\left(t_{i}\right)\right)\right]\right\|^{2} \\
& +5 \mathbb{E}\left\|\sum_{t_{i}<t} K_{q}\left(t-t_{i}\right)\left[J_{i}\left(y\left(t_{i}\right)+\widehat{z}_{n}\left(t_{i}\right)\right)-J_{i}\left(y\left(t_{i}\right)+\widehat{z}\left(t_{i}\right)\right)\right] d s\right\|^{2} \\
& +5 \mathbb{E} \| \int_{0}^{t} R_{q}(t-s) B B^{*} R_{q}^{*}(T-s) R\left(\lambda, \Gamma_{0}^{T}\right)\left\{\left(\widehat{z}_{n}\right)_{T}-\widehat{z}_{T}-S_{q}(t)\left[g\left(y+\widehat{z}_{n}\right)\right.\right. \\
& -g(y+\widehat{z})]-K_{q}(t)\left[h\left(y+\widehat{z}_{n}\right)-h(y+\widehat{z})\right]-\sum_{i=1}^{\infty} S_{q}\left(T-t_{i}\right)\left[I_{i}\left(y\left(t_{i}\right)+\widehat{z}_{n}\left(t_{i}\right)\right)\right. \\
& \left.-I_{i}\left(y\left(t_{i}\right)+\widehat{z}\left(t_{i}\right)\right)\right]-\sum_{t_{i}<t} K_{q}\left(t-t_{i}\right)\left[J_{i}\left(y\left(t_{i}\right)+\widehat{z}_{n}\left(t_{i}\right)\right)-J_{i}\left(y\left(t_{i}\right)+\widehat{z}\left(t_{i}\right)\right)\right] d s \\
& -\int_{0}^{T} K_{q}(T-s)\left[G\left(s, y_{s}+\left(\widehat{z}_{n}\right)_{s}, \int_{0}^{s} a_{1}\left(s, \zeta, y_{\zeta}+\left(\widehat{z}_{n}\right)_{\zeta}\right) d \zeta\right)\right.
\end{aligned}
$$




$$
\begin{aligned}
& \left.-G\left(s, y_{s}+\widehat{z}_{s}, \int_{0}^{s} a_{1}\left(s, \zeta, y_{\zeta}+\widehat{z}_{t}\right) d \zeta\right)\right] d s-\int_{0}^{T} R_{q}(T-s)\left[F \left(s, y_{s}+\left(\widehat{z}_{n}\right)_{s},\right.\right. \\
& \left.\left.\int_{0}^{s} a_{2}\left(s, \zeta, y_{\zeta}+\left(\widehat{z}_{n}\right)_{\zeta}\right) d \zeta\right)-F\left(s, y_{s}+\widehat{z}_{s}, \int_{0}^{s} a_{2}\left(s, \zeta, y_{\zeta}+\widehat{z}_{\zeta}\right) d \zeta\right)\right] d s \\
& -\int_{0}^{T} R_{q}(T-s)\left[H\left(s, y_{s}+\left(\widehat{z}_{n}\right)_{s}, \int_{0}^{s} a_{3}\left(s, \zeta, y_{\zeta}+\left(\widehat{z}_{n}\right)_{\zeta}\right) d \zeta\right)\right. \\
& \left.\left.\quad-H\left(s, y_{s}+\widehat{z}_{s}, \int_{0}^{s} a_{3}\left(s, \zeta, y_{\zeta}+\widehat{z}_{t}\right) d \zeta\right)\right] d W(s)\right\} d s\left\|^{2}+5 \mathbb{E}\right\| \int_{0}^{t} R_{q}(t-s) \\
& \times\left[F\left(s, y_{s}+\left(\widehat{z}_{n}\right)_{s}, \int_{0}^{s} a_{2}\left(s, \zeta, y_{\zeta}+\left(\widehat{z}_{n}\right)_{\zeta}\right) d \zeta\right)\right. \\
& \left.-F\left(s, y_{s}+\widehat{z}_{s}, \int_{0}^{s} a_{2}\left(s, \zeta, y_{\zeta}+\widehat{z}_{\zeta}\right) d \zeta\right)\right] d s \|^{2} \\
& +5 \mathbb{E} \| \int_{0}^{t} \mathcal{R}_{\alpha}(t-s)\left[H\left(s, y_{s}+\left(\widehat{z}_{n}\right)_{s}, \int_{0}^{s} a_{3}\left(s, \zeta, y_{\zeta}+\left(\widehat{z}_{n}\right)_{\zeta}\right) d \zeta\right)\right. \\
& \left.\left.\quad-H\left(s, y_{s}+\widehat{z}_{s}, \int_{0}^{s} a_{3}\left(s, \zeta, y_{\zeta}+\widehat{z}_{\zeta}\right) d \zeta\right)\right]\right) d W(s) \|^{2} \rightarrow 0, \text { as } n \rightarrow \infty .
\end{aligned}
$$

Step 4. $\Psi_{2}$ maps bounded sets into equicontinuous sets of $\mathcal{B}_{r}$.

Let $\tau_{1}, \tau_{2} \in\left(t_{i}, t_{i+1}\right], i=1, \cdots, m$ with $\tau_{2}>\tau_{1}$. For $z \in \mathcal{B}_{r}$ $\mathbb{E}\left\|\Psi_{2} z\left(\tau_{2}\right)-\Psi_{2} z\left(\tau_{1}\right)\right\|_{E}^{2}$

$$
\begin{aligned}
\leq & 5 \mathbb{E}\left\|\sum_{0<t_{i}<t}\left[S_{q}\left(\tau_{2}-t_{i}\right)-S_{q}\left(\tau_{1}-t_{i}\right)\right] I_{i}\left(y\left(t_{i}\right)+\widehat{z}\left(t_{i}\right)\right)\right\|^{2} \\
& +5 \mathbb{E}\left\|\sum_{0<t_{i}<t}\right\|\left[K_{q}\left(\tau_{2}-t_{i}\right)-K_{q}\left(\tau_{1}-t_{i}\right)\right] J_{i}\left(y\left(t_{i}\right)+\widehat{z}\left(t_{i}\right)\right) \|^{2} \\
& +5 \mathbb{E}\left\|\int_{0}^{\tau_{2}} R_{q}\left(\tau_{2}-s\right) B x(s) d s-\int_{0}^{\tau_{1}} R_{q}\left(\tau_{1}-s\right) B x(s) d s\right\|^{2} \\
& +5 \mathbb{E} \| \int_{0}^{\tau_{2}} R_{q}\left(\tau_{2}-s\right) F\left(s, y_{s}+\widehat{z}_{s}, \int_{0}^{s} a_{2}\left(s, \zeta, y_{\zeta}+\widehat{z}_{\zeta}\right) d \zeta\right) d s-\int_{0}^{\tau_{1}} R_{q}\left(\tau_{1}-s\right) \\
& \times F\left(s, y_{s}+\widehat{z}_{s}, \int_{0}^{s} a_{2}\left(s, \zeta, y_{\zeta}+\widehat{z}_{\zeta}\right) d \zeta\right) d s\left\|^{2}+5 \mathbb{E}\right\| \int_{0}^{\tau_{2}} R_{q}\left(\tau_{2}-s\right) \\
& \times H\left(s, y_{s}+\widehat{z}_{s}, \int_{0}^{s} a_{3}\left(s, \zeta, y_{\zeta}+\widehat{z}_{\zeta}\right) d \zeta\right) d W(s)-\int_{0}^{\tau_{1}} R_{q}\left(\tau_{1}-s\right) \\
& \times H\left(s, y_{s}+\widehat{z}_{s}, \int_{0}^{s} a_{3}\left(s, \zeta, y_{\zeta}+\widehat{z}_{\zeta}\right) d \zeta\right) d W(s) \|^{2} \\
\leq & 5 \sum_{0<t_{i}<t} \mathbb{E}\left\|S_{q}\left(\tau_{2}-t_{i}\right)-S_{q}\left(\tau_{1}-t_{i}\right)\right\|^{2} \cdot\left\|I_{i}\left(y\left(t_{i}\right)+\widehat{z}\left(t_{i}\right)\right)\right\|^{2} \\
& +5 \sum_{0<t_{i}<t} \mathbb{E}\left\|K_{q}\left(\tau_{2}-t_{i}\right)-K_{q}\left(\tau_{1}-t_{i}\right)\right\|^{2}\left\|J_{i}\left(y\left(t_{i}\right)+\widehat{z}\left(t_{i}\right)\right)\right\|^{2}+10 M^{2} M_{B}^{2}\left(\tau_{2}-\tau_{1}\right) \\
& \times \int_{\tau_{1}}^{\tau_{2}} \mathbb{E}\|x(s)\|^{2} d s+10 \int_{0}^{\tau_{1}}\left\|R_{q}\left(\tau_{2}-s\right)-R_{q}\left(\tau_{1}-s\right)\right\| d s
\end{aligned}
$$




$$
\begin{aligned}
& \times \int_{0}^{\tau_{1}}\left\|R_{q}\left(\tau_{2}-s\right)-R_{q}\left(\tau_{1}-s\right)\right\| \mathbb{E}\|B x(s)\|^{2} d s+10 M^{2}\left(\tau_{2}-\tau_{1}\right) \\
& \times \int_{\tau_{1}}^{\tau_{2}} \mathbb{E}\left\|F\left(s, y_{s}+\widehat{z}_{s}, \int_{0}^{s} a_{2}\left(s, \zeta, y_{\zeta}+\widehat{z}_{\zeta}\right) d \zeta\right)\right\|^{2} d s \\
& +10 \int_{0}^{\tau_{1}}\left\|R_{q}\left(\tau_{2}-s\right)-R_{q}\left(\tau_{1}-s\right)\right\| d s \\
& \times \int_{0}^{\tau_{1}}\left\|R_{q}\left(\tau_{2}-s\right)-R_{q}\left(\tau_{1}-s\right)\right\| \mathbb{E}\left\|F\left(s, y_{s}+\widehat{z}_{s}, \int_{0}^{s} a_{2}\left(s, \zeta, y_{\zeta}+\widehat{z}_{\zeta}\right) d \zeta\right)\right\|^{2} d s \\
& +12 T r(\mathcal{Q}) \\
& \times \int_{\tau_{1}}^{\tau_{2}} \mathbb{E}\left\|R_{q}(t-s) H\left(s, y_{s}+\widehat{z}_{s}, \int_{0}^{s} a_{3}\left(s, \zeta, y_{\zeta}+\widehat{z}_{\zeta}\right) d \zeta\right)\right\|^{2} d s+10 T r(\mathcal{Q}) \\
& \times \int_{0}^{\tau_{1}}\left\|R_{q}\left(\tau_{2}-s\right)-R_{q}\left(\tau_{1}-s\right)\right\|^{2} \mathbb{E}\left\|H\left(s, y_{s}+\widehat{z}_{s}, \int_{0}^{s} a_{3}\left(s, \zeta, y_{\zeta}+\widehat{z}_{\zeta}\right) d \zeta\right)\right\|^{2} d s
\end{aligned}
$$

Thus compactness of $S_{q}(\cdot), K_{q}(\cdot)$ and $R_{q}(\cdot)$ gives the continuity in the uniform operator topology which implies that the above inequality tends to zero as $\tau_{1} \rightarrow \tau_{2}$. This implies that the set $\left\{\Psi_{2} z: z \in \mathcal{B}_{r}\right\}$ is a family of equicontinuous functions.

Step 5. $\Psi_{2}$ maps $\mathcal{B}_{r}$ into a precompact subset of $\mathcal{B}_{T}^{0}$.

Obviously, the set $\mathcal{V}(0)=\left\{\Psi_{2}(0)\right\}$ is relatively compact in $E$. For $t \in(0, T]$, we decompose the $\Psi_{2}$ by $\Psi_{2}=\Lambda_{1}+\Lambda_{2}$ as

$$
\begin{aligned}
\Lambda_{1} z(t) & =\int_{0}^{t} R_{q}(t-s) B x(s) d s+\int_{0}^{t} R_{q}(t-s) F\left(s, y_{s}+\widehat{z}_{s}, \int_{0}^{s} a_{2}\left(s, \zeta, y_{\zeta}+\widehat{z}_{\zeta}\right) d \zeta\right) d s \\
& +\int_{0}^{t} R_{q}(t-s) H\left(s, y_{s}+\widehat{z}_{s}, \int_{0}^{s} a_{3}\left(s, \zeta, y_{\zeta}+\widehat{z}_{\zeta}\right) d \zeta\right) d W(s), \quad t \in[0, T], \quad \text { (3.14) }
\end{aligned}
$$

and

$$
\begin{array}{r}
\Lambda_{2} z(t)=\sum_{i=1}^{m} S_{q}\left(t-t_{i}\right) I_{i}\left(y\left(t_{i}\right)+\widehat{z}\left(t_{i}\right)\right)+\sum_{i=1}^{m} K_{q}\left(t-t_{i}\right) J_{i}\left(y\left(t_{i}\right)+\widehat{z}\left(t_{i}\right)\right) d s \\
t \in(0, T] .
\end{array}
$$

Now, it will be shown that $\Lambda_{1}\left(\mathcal{B}_{r}\right)(t)=\left\{\left(\Lambda_{1} z\right)(t): z \in \mathcal{B}_{r}\right\}$ is relatively compact for every $t \in[0, T]$. Let $0<t \leq s \leq t_{1}$ be fixed and let $\epsilon$ be a positive real number such that $\epsilon<t$. For $z \in \mathcal{B}_{r}$, we consider

$$
\begin{aligned}
\left(\Lambda_{1}^{\epsilon} z\right)(t) & =\int_{0}^{t-\epsilon} R_{q}(t-s) B x(s) d s+\int_{0}^{t-\epsilon} R_{q}(t-s) F\left(s, y_{s}+\widehat{z}_{s}, \int_{0}^{s} a_{2}\left(s, \zeta, y_{\zeta}+\widehat{z}_{\zeta}\right) d \zeta\right) d s \\
& +\int_{0}^{t-\epsilon} R_{q}(t-s) H\left(s, y_{s}+\widehat{z}_{s}, \int_{0}^{s} a_{3}\left(s, \zeta, y_{\zeta}+\widehat{z}_{\zeta}\right) d \zeta\right) d W(s), \quad t \in[0, T] .
\end{aligned}
$$

By the compactness of $S_{q}(t), R_{q}(t) t>0$, we have that the set $U_{\epsilon}(t)=\left\{\left(\Lambda_{1}^{\epsilon} z\right)(t): z \in\right.$ $\left.\mathcal{B}_{r}\right\}$ is relatively compact in $E$ for each $\epsilon$ with $\epsilon \in(0, t)$. Thus, we have $\mathbb{E} \|\left(\Lambda_{1} z\right)(t)-$ 


$$
\begin{aligned}
\left(\Lambda_{1}^{\epsilon} z\right)(t) & \|_{E}^{2} \\
\leq & 3 \mathbb{E}\left\|\int_{t-\epsilon}^{t} R_{q}(t-s) B x(s) d s\right\|^{2} \\
& +3 \mathbb{E}\left\|\int_{t-\epsilon}^{t} R_{q}(t-s) F\left(s, y_{s}+\widehat{z}_{s}, \int_{0}^{s} a_{2}\left(s, \zeta, y_{\zeta}+\widehat{z}_{\zeta}\right) d \zeta\right) d s\right\|^{2} \\
& +3 \mathbb{E}\left\|\int_{t-\epsilon}^{t} R_{q}(t-s) H\left(s, y_{s}+\widehat{z}_{s}, \int_{0}^{s} a_{3}\left(s, \zeta, y_{\zeta}+\widehat{z}_{\zeta}\right) d \zeta\right) d W(s)\right\|^{2} \\
\leq \quad & 3 M^{2} M_{B}^{2} \mathbb{O} \epsilon^{2}+3 M^{2} \epsilon^{2} \int_{t-\epsilon}^{t} m_{F}(s) \Theta_{F}\left(4\left[\|\phi\|_{\mathfrak{B}_{v}}^{2}+l^{2}\left(r+M^{2} \mathbb{E}\|\phi(0)\|_{E}^{2}\right)\right]\right. \\
& \left.+T \int_{0}^{s} L_{a_{2}} \mathcal{W}_{a_{2}}\left(4\left[\|\phi\|_{\mathfrak{B}_{v}}^{2}+l^{2}\left(r+M^{2} \mathbb{E}\|\phi(0)\|_{E}^{2}\right)\right]\right) d \zeta\right) d s+3 M^{2} \operatorname{Tr}(\mathcal{Q}) \epsilon \\
& \times \int_{t-\epsilon}^{t} m_{H}(s) \Theta_{H}\left(4\left[\|\phi\|_{\mathfrak{B}_{v}}^{2}+l^{2}\left(r+M^{2} \mathbb{E}\|\phi(0)\|_{E}^{2}\right)\right]+T \int_{0}^{s} m_{a_{3}} \Theta_{a_{3}}\left(4 \left[\|\phi\|_{\mathfrak{B}_{v}}^{2}\right.\right.\right. \\
& \left.\left.\left.+l^{2}\left(r+M^{2} \mathbb{E}\|\phi(0)\|_{E}^{2}\right)\right]\right) d \zeta\right) d s .
\end{aligned}
$$

As $\epsilon \rightarrow 0$, the right hand side of above inequality tends to zero. Thus, there are relatively compact sets arbitrary close to the set $U(t)=\left\{\left(\Lambda_{1} z\right)(t): z \in \mathcal{B}_{r}\right\}$ and $U(t)$ is relatively compact in $E$. It is not difficult to show that $\Lambda_{1}\left(\mathcal{B}_{r}\right)$ is uniformly bounded. Since $\Psi_{2}$ is equicontinuous. Thus, by the Arzelá-Ascoli theorem, we deduce that $\Lambda_{1}$ is compact.

Next, we show that $\Lambda_{2}\left(\mathcal{B}_{q}\right)(t)$ is relatively compact for every $t \in[0, T]$. For $t \in\left[0, t_{1}\right]$, it is obvious. Now for $t \in\left(t_{i}, t_{i+1}\right], i=1, \cdots, m$ and $z \in \mathcal{B}_{r}$, we need to show that $U=\left\{\sum_{i=1}^{m} S_{q}\left(t-t_{i}\right) I_{i}\left(y\left(t_{i}\right)+\widehat{z}\left(t_{i}\right)\right)+\sum_{i=1}^{m} K_{q}\left(t-t_{i}\right) J_{i}\left(y\left(t_{i}\right)+\widehat{z}\left(t_{i}\right)\right) d s\right.$ : $\left.t \in\left(t_{i}, t_{i+1}\right], z \in \mathcal{B}_{r}\right\}$ is relatively compact in $C\left(\left[t_{i}, t_{i+1}\right] ; E\right)$. By the compactness of $S_{q}(t), K_{q}(t) t \geq 0$ and assumptions on $I_{i}, J_{i}$, we conclude that the set $\left\{\sum_{i=1}^{m} S_{q}(t-\right.$ $\left.\left.t_{i}\right) I_{i}\left(y\left(t_{i}\right)+\widehat{z}\left(t_{i}\right)\right)+\sum_{i=1}^{m} K_{q}\left(t-t_{i}\right) J_{i}\left(y\left(t_{i}\right)+\widehat{z}\left(t_{i}\right)\right) d s, z \in \mathcal{B}_{r}\right\}$ is relatively compact in $E$. It can be easily prove that the functions in $U$ are equicontinuous. Thus, from the Arzelá-Ascoli theorem, it follows that $\Lambda_{2}$ is compact operator. Hence, $\Psi_{2}=\Lambda_{1}+\Lambda_{2}$ is completely continuous operator.

Step 6. The set $\mathcal{G}=\left\{u \in E: \lambda_{1} \Psi_{1}\left(u / \lambda_{1}\right)+\lambda_{1} \Psi_{2} u=u\right\}$ is bounded for $\lambda_{1} \in(0,1)$. Consider the nonlinear operator equation of the form

$$
z(t)=\lambda_{1} \Psi z(t), \quad 0<\lambda_{1}<1
$$

where $\Psi$ is defined by the equation (3.7).

Let $z \in \mathfrak{B}_{T}^{0}$ be a possible solution of equation (3.16) that gives that

$$
\begin{aligned}
z(t)= & \lambda_{1} S_{q}(t)[-g(y+\widehat{z})]+\lambda_{1} K_{q}(t)\left[u_{1}-h(y+\widehat{z})\right]+\lambda_{1} \sum_{0<t_{i}<t} S_{q}\left(t-t_{i}\right) \\
& \times I_{i}\left(y\left(t_{i}\right)+\widehat{z}\left(t_{i}\right)\right)+\lambda_{1} \sum_{t_{i}<t} K_{q}\left(t-t_{i}\right) J_{i}\left(y\left(t_{i}\right)+\widehat{z}\left(t_{i}\right)\right)+\lambda_{1} \int_{0}^{t} K_{q}(t-s)
\end{aligned}
$$




$$
\begin{aligned}
& \times G\left(s, y_{s}+\widehat{z}_{s}, \int_{0}^{s} a_{1}\left(s, \zeta, y_{\zeta}+\widehat{z}_{\zeta}\right) d \zeta\right) d s+\lambda_{1} \int_{0}^{t} R_{q}(t-s) B x(s) d s \\
& +\lambda_{1} \int_{0}^{t} R_{q}(t-s) F\left(s, y_{s}+\widehat{z}_{s}, \int_{0}^{s} a_{2}\left(s, \zeta, y_{\zeta}+\widehat{z}_{\zeta}\right) d \zeta\right) d s \\
& +\lambda_{1} \int_{0}^{t} R_{q}(t-s) H\left(s, y_{s}+\widehat{z}_{s}, \int_{0}^{s} a_{3}\left(s, \zeta, y_{\zeta}+\widehat{z}_{\zeta}\right) d \zeta\right) d W(s), \\
& t \in[0, T] .
\end{aligned}
$$

Let $\nu(t)=4\left(\|\phi\|_{\mathfrak{B}_{v}}^{2}+l^{2}\left(r+M_{\mathcal{S}}^{2} \mathbb{E}\|\phi(0)\|_{E}^{2}\right)\right)$ for each $t \in[0, T]$. By using assumptions $(A 2)-(A 5)$, we get

$$
\begin{aligned}
& \mathbb{E}\|z(t)\|^{2} \leq 8 \mathbb{E}\left\|S_{q}(t)[-g(y+\widehat{z})]\right\|^{2} \\
& +8 \mathbb{E}\left\|K_{q}(t)\left[u_{1}-h(y+\widehat{z})\right]\right\|^{2}+8 \mathbb{E} \| \sum_{0<t_{i}<t} S_{q}\left(t-t_{i}\right) \\
& \times I_{i}\left(y\left(t_{i}\right)+\widehat{z}\left(t_{i}\right)\right)\left\|^{2}+\mathbb{E}\right\| \sum_{t_{i}<t} K_{q}\left(t-t_{i}\right) J_{i}\left(y\left(t_{i}\right)+\widehat{z}\left(t_{i}\right)\right)\left\|^{2}+8 \mathbb{E}\right\| \int_{0}^{t} K_{q}(t-s) \\
& \times G\left(s, y_{s}+\widehat{z}_{s}, \int_{0}^{s} a_{1}\left(s, \zeta, y_{\zeta}+\widehat{z}_{\zeta}\right) d \zeta\right) d s\left\|^{2}+8 \mathbb{E}\right\| \int_{0}^{t} R_{q}(t-s) B x(s) d s \|^{2} \\
& +8 \mathbb{E}\left\|\int_{0}^{t} R_{q}(t-s) F\left(s, y_{s}+\widehat{z}_{s}, \int_{0}^{s} a_{2}\left(s, \zeta, y_{\zeta}+\widehat{z}_{\zeta}\right) d \zeta\right) d s\right\|^{2} \\
& +8 \mathbb{E}\left\|\int_{0}^{t} R_{q}(t-s) H\left(s, y_{s}+\widehat{z}_{s}, \int_{0}^{s} a_{3}\left(s, \zeta, y_{\zeta}+\widehat{z}_{\zeta}\right) d \zeta\right) d W(s)\right\|^{2}, \\
& \leq 8 M^{2}\|-g(y+\widehat{z})\|^{2}+8 M^{2} \sum_{i=1}^{m} \Phi_{1}^{i}+8 M^{2} \sum_{i=1}^{m} \Psi_{2}^{i}+64 \frac{M^{4} M_{B}^{4} T^{2}}{\lambda^{2}}\left\{2 \mathbb{E}\left\|y_{T}+\widehat{z}_{T}\right\|^{2}\right. \\
& +2 \int_{0}^{T} \mathbb{E}\|\sigma(s)\|_{\mathcal{Q}^{2}}^{2} d s+2 M^{2}\left[\|\phi\|_{\mathfrak{B}_{v}}^{2}+\widehat{L}_{g}\|y+\widehat{z}\|^{2}+\widehat{L}_{g}^{1}\right]+2 M^{2}\left[\left\|u_{1}\right\|^{2}\right. \\
& \left.+\widehat{L}_{h}\|y+\widehat{z}\|^{2}+\widehat{L}_{h}^{1}\right] \\
& +M^{2} \sum_{i=1}^{m} \Phi_{1}^{i}+M^{2} \sum_{i=1}^{m} \Psi_{2}^{i}+M^{2} T^{2}\left[2 L_{G}\left(1+2 L_{a_{1}}\right) r^{*}+4 L_{G} \mathcal{C}_{2}+2 \mathcal{C}_{1}\right] \\
& +M^{2} T \int_{0}^{T} m_{F}(s) \Theta_{F}\left(\left\|y_{s}+\widehat{z}_{s}\right\|_{\mathfrak{B}_{v}}^{2}+T \int_{0}^{s} L_{a_{2}} \mathcal{W}_{a_{2}}\left(\left\|y_{\zeta}+\widehat{z}_{\zeta}\right\|_{\mathfrak{B}_{v}}^{2}\right) d \zeta\right) d s+M^{2} \operatorname{Tr}(\mathcal{Q}) \\
& \left.\times \int_{0}^{T} m_{H}(s) \Theta_{H}\left(\left\|y_{s}+\widehat{z}_{s}\right\|_{\mathfrak{B}_{v}}^{2}+T \int_{0}^{s} m_{a_{3}} \Theta_{a_{3}}\left(\left\|y_{\zeta}+\widehat{z}_{\zeta}\right\|_{\mathfrak{B}_{v}}^{2}\right) d \zeta\right) d s\right\}+8 M T \\
& \times \int_{0}^{t}\left\|R_{q}(t-s)\right\| m_{F}(s) \Theta_{F}\left(\left\|y_{s}+\widehat{z}_{s}\right\|_{\mathfrak{B}_{v}}^{2}+T \int_{0}^{s} L_{a_{2}} \mathcal{W}_{a_{2}}\left(\left\|y_{\zeta}+\widehat{z}_{\zeta}\right\|_{\mathfrak{B}_{v}}^{2}\right) d \zeta\right) d s \\
& +8 M^{2} \operatorname{Tr}(\mathcal{Q}) \int_{0}^{t} m_{H}(s) \Theta_{H}\left(\left\|y_{s}+\widehat{z}_{s}\right\|_{\mathfrak{B}_{v}}^{2}+T \int_{0}^{s} m_{a_{3}} \Theta_{a_{3}}\left(\left\|y_{\zeta}+\widehat{z}_{\zeta}\right\|_{\mathfrak{B}_{v}}^{2}\right) d \zeta\right) d s \\
& \leq 16 M^{2}\left[\widehat{L}_{g}\left\|y_{t}+\widehat{z}_{t}\right\|_{\mathfrak{B}_{v}}^{2}+\widehat{L}_{g}^{1}\right]+16 M^{2}\left[\left\|u_{1}\right\|^{2}+\widehat{L}_{h}\left\|y_{t}+\widehat{z}_{t}\right\|_{\mathfrak{B}_{v}}^{2}+\widehat{L}_{h}^{1}\right]+8 M^{2} \sum_{i=1}^{m} \Phi_{1}^{i}
\end{aligned}
$$




$$
\begin{aligned}
& +8 M^{2} \sum_{i=1}^{m} \Psi_{2}^{i}+64 \frac{M^{4} M_{B}^{4} T^{2}}{\lambda^{2}} \times\left\{2 \mathbb{E}\left\|y_{T}+\widehat{z}_{T}\right\|^{2}+2 \int_{0}^{T} \mathbb{E}\|\sigma(s)\|_{\mathcal{Q}}^{2} d s+2 M^{2}\left[\|\phi\|_{\mathfrak{B}_{v}}^{2}\right.\right. \\
& \left.+\widehat{L}_{g}\left\|y_{t}+\widehat{z}_{t}\right\|_{\mathfrak{B}_{v}}^{2}+\widehat{L}_{g}^{1}\right]+2 M^{2}\left[\left\|u_{1}\right\|_{E}^{2}+\widehat{L}_{h}\|y+\widehat{z}\|^{2}+\widehat{L}_{h}^{1}\right]+M^{2} \sum_{i=1}^{m} \Phi_{1}^{i}+M^{2} \sum_{i=1}^{m} \Psi_{2}^{i} \\
& +M^{2} T^{2}\left[2 L_{G}\left(1+2 L_{a_{1}}\right) \nu(t)+4 L_{G} \mathcal{C}_{2}+2 \mathcal{C}_{1}\right]+M^{2} T^{2} \int_{0}^{T} m_{F}(s) \\
& \times \Theta_{F}\left(\nu(s)+T \int_{0}^{s} L_{a_{2}} \mathcal{W}_{a_{2}}(\nu(\zeta)) d \zeta\right) d s+M^{2} \operatorname{Tr}(\mathcal{Q}) T \int_{0}^{T} m_{H}(s) \Theta_{H}(\nu(s) \\
& \left.\left.+T \int_{0}^{s} m_{a_{3}} \Theta_{a_{3}}(\nu(\zeta)) d \zeta\right) d s\right\}+8 M^{2} T^{2}\left[2 L_{G}\left(1+2 L_{a_{1}}\right) \nu(t)+4 L_{G} \mathcal{C}_{2}+2 \mathcal{C}_{1}\right] \\
& +8 M^{2} T^{2} \int_{0}^{t} m_{F}(s) \Theta\left(\nu(s)+T \int_{0}^{s} L_{a_{2}} \mathcal{W}_{a_{2}}(\nu(\zeta)) d \zeta\right) d s+8 M^{2} \operatorname{Tr}(\mathcal{Q}) T \\
& \times \int_{0}^{t} m_{H}(s) \Theta_{H}\left(\nu(s)+T \int_{0}^{s} m_{a_{3}} \Theta_{a_{3}}(\nu(\zeta)) d \zeta\right) d s .
\end{aligned}
$$

Therefore

$$
\begin{aligned}
\nu(t) \leq & \frac{\widetilde{M}}{1-\widetilde{N}}+\frac{32 T^{2} l^{2} M^{2}}{1-\widetilde{N}} \times \int_{0}^{t} m_{F}(s) \Theta_{F}\left(\nu(s)+T \int_{0}^{s} L_{a_{2}} \mathcal{W}_{a_{2}}(\nu(\zeta)) d \zeta\right) d s \\
& +\frac{32 l^{2} M^{2} \operatorname{Tr}(Q) T}{(1-\widetilde{N})} \int_{0}^{t} m_{H}(s) \Theta_{H}\left(\nu(s)+T \int_{0}^{s} m_{a_{3}} \Theta_{a_{3}}(\nu(\zeta)) d \zeta\right) d s
\end{aligned}
$$

where

$$
\begin{aligned}
& \widetilde{M}=4\left(\|\phi\|_{\mathfrak{B}_{v}}^{2}+M^{2} l^{2} \mathbb{E}\|\phi(0)\|^{2}\right)+64 M^{2} l^{2} \widehat{L}_{g}^{1}+64 M^{2} l^{2} \widehat{L}_{h}^{1}+64 l^{2} M^{2}\left(\left\|u_{1}\right\|_{E}^{2}\right) \\
& +32 l^{2} M^{2} \sum_{i=1}^{m} \Phi_{1}^{i}+32 l^{2} M^{2} \sum_{i=1}^{m} \Psi_{2}^{i}+256 l^{2} \frac{M^{4} M_{B}^{4} T^{4}}{\lambda^{2}} \times\left\{2 \mathbb{E}\left\|y_{T}+\widehat{z}_{T}\right\|^{2}\right. \\
& +2 \int_{0}^{T} \mathbb{E}\|\sigma(s)\|_{\mathcal{Q}}^{2} d s+2 M^{2}\|\phi\|_{\mathfrak{B}_{v}}^{2}+2 M^{2} \widehat{L}_{g}^{1}+2 M^{2} \widehat{L}_{h}^{1}+2 M^{2}\left\|u_{1}\right\|_{E}^{2}+M^{2} \sum_{i=1}^{m} \Phi_{1}^{i} \\
& +M^{2} \sum_{i=1}^{m} \Psi_{2}^{i}+2 M^{2} T^{2}\left(2 L_{G} \mathcal{C}_{2}+\mathcal{C}_{1}\right)+M^{2} T^{2} \int_{0}^{T} m_{F}(\tau) \Theta_{F}(\nu(\tau) \\
& \left.+T \int_{0}^{\tau} L_{a_{2}} \mathcal{W}_{a_{2}}(\nu(\zeta)) d \zeta\right) d \tau+M^{2} \operatorname{Tr}(\mathcal{Q}) T \int_{0}^{T} m_{H}(\tau) \Theta_{H}(\nu(\tau) \\
& \left.\left.+T \int_{0}^{\tau} m_{a_{3}} \Theta_{a_{3}}(\nu(\zeta)) d \zeta\right) d s\right\}+16 M^{2} T^{2}\left(2 L_{G} \mathcal{C}_{2}+\mathcal{C}_{1}\right)
\end{aligned}
$$

and

$$
\widetilde{N}=64 l^{2} M^{2} \widehat{L}_{g}+64 M^{2} l^{2} \widehat{L}_{h}+8 l^{2}\left(\frac{64 M^{4} M_{B}^{4} T^{4}}{\lambda^{2}}+8 M^{2} T^{2}\right) L_{G}\left(1+2 L_{a_{1}}\right)
$$




$$
+\frac{256 M^{4} M_{B}^{2} T^{4}}{\lambda^{2}} l^{2} \times\left\{2 M^{2} \widehat{L}_{g}+2 M^{2} \widehat{L}_{h}\right\} .
$$

Denote the right hand side of the inequality (3.18) by $\xi$ and obtain

$$
\nu(t) \leq \xi(t), \quad \forall \quad t \in[0, T]
$$

with $\xi(0)=\frac{\widetilde{M}}{1-\widetilde{N}}$. Therefore, we have

$$
\begin{aligned}
\xi^{\prime}(t)= & \frac{32}{(1-\widetilde{N})}\left[l^{2} M^{2} T^{2} \times m_{F}(t) \Theta_{F}\left(\nu(t)+T \int_{0}^{t} L_{a_{2}} \mathcal{W}_{a_{2}}(\nu(s)) d s\right)\right. \\
& \left.+l^{2} M^{2} \operatorname{Tr}(Q) T \times m_{H}(t) \Theta_{H}\left(\nu(t)+T \int_{0}^{t} m_{a_{3}} \Theta_{a_{3}}(\nu(s)) d s\right)\right], \\
\leq & \frac{32}{(1-\widetilde{N})}\left[l^{2} M^{2} T^{2} \times m_{F}(t) \Theta_{F}\left(\xi(t)+T \int_{0}^{t} L_{a_{2}} \mathcal{W}_{a_{2}}(\xi(s)) d s\right)\right. \\
& \left.+l^{2} M^{2} \operatorname{Tr}(Q) T \times m_{H}(t) \Theta_{H}\left(\xi(t)+T \int_{0}^{t} m_{a_{3}} \Theta_{a_{3}}(\xi(s)) d s\right)\right] \\
\leq & \bar{m}(t)\left[\Theta_{F}\left(\xi(t)+T \int_{0}^{t} L_{a_{2}} \mathcal{W}_{a_{2}}(\xi(s)) d s\right)\right. \\
& \left.+\Theta_{H}\left(\xi(t)+T \int_{0}^{t} m_{a_{3}} \Theta_{a_{3}}(\xi(s)) d s\right)\right]
\end{aligned}
$$

where $\bar{m}(t)=\max \left\{\frac{32}{(1-\widetilde{N})} \times l^{2} M^{2} T^{2} \times m_{F}(t), \frac{32}{(1-\widetilde{N})} l^{2} M^{2} \operatorname{Tr}(Q) T \times m_{H}(t)\right\}$. Let us consider $\varphi(t)=\xi(t)+\int_{0}^{t} T \mathfrak{L} \Theta(\xi(s)) d s$, where $\mathfrak{L}=\max \left\{L_{a_{2}}, m_{a_{3}}\right\}$, and $\Theta(y)=$ $\max \left\{\mathcal{W}_{a_{2}}(y), \Theta_{a_{3}}(y)\right\}$. Thus, $\varphi(0)=\xi(0), \xi(t) \leq \varphi(t)$ and

$$
\begin{aligned}
\varphi^{\prime}(t) & =\xi^{\prime}(t)+T \mathfrak{L} \Theta(\xi(t)), \\
& \leq \bar{m}(t)\left[\Theta_{F}(\varphi(t))+\Theta_{H}(\varphi(t))\right]+T \mathfrak{L} \Theta(\varphi(t)), \\
& \leq \widehat{m}(t)\left[\Theta_{F}(\varphi(t))+\Theta_{H}(\varphi(t))+\Theta(\varphi(t))\right],
\end{aligned}
$$

where $\widehat{m}(t)=\max \{\bar{m}(t), T \mathfrak{L}\}$. This implies that

$$
\int_{\varphi(0)}^{\varphi(t)} \frac{d s}{\Theta_{F}(s)+\Theta_{H}(s)+\Theta(s)} \leq \int_{0}^{T} \widehat{m}(s) d s \leq \int_{\xi(0)}^{\infty} \frac{d s}{\Theta_{F}(s)+\Theta_{H}(s)+\Theta(s)}
$$

which shows that $\varphi(t)$ is bounded on $[0, T]$. Therefore, there exists a constant $\mathfrak{C}>0$ such that $\|u\|_{T}^{2} \leq \nu(t) \leq \xi(t) \leq \varphi(t) \leq \mathfrak{C}$ for all $t \in[0, T]$, where constant $\mathfrak{C}$ depends on the function $\Theta_{F}, \Theta_{H}, \Theta, m_{F}, m_{H}, \bar{m}$ and $\widehat{m}$. Therefore, it implies that the set $\mathcal{G}$ is bounded on $[0, T]$. Hence, by the Krasnoselskii-Schaefer type fixed point theorem, there exists a fixed point $z$ for $\Psi$ on $\mathcal{B}_{r}$ such that $\Psi z(t)=z(t)$. Since $u(t)=y(t)+\widehat{z}(t)$, therefore $u(t)$ is the mild solution for the problem (1.1)-(1.3) on $[0, T]$. 


\section{APPROXIMATE CONTROLLABILITY}

This section presents the main result on approximate controllability of system (1.1)(1.3). For this, we have to make the following assumptions:

(B1) The function $G:[0, T] \rightarrow \mathfrak{B}_{v} \times E \rightarrow E$ is continuous, and there exists a constant $\widetilde{C}_{1}>0$ such that

$$
\mathbb{E}\left\|G\left(t, u_{1}, u_{2}\right)\right\|^{2} \leq \widetilde{C}_{1},
$$

for $t \in[0, T]$ and $u_{1} \in \mathfrak{B}_{v}, u_{2} \in E$.

(B2) There exists a constant $\widetilde{C}_{2}>0$ such that

$$
\mathbb{E}\left\|F\left(t, u_{1}, u_{2}\right)\right\|^{2} \leq \widetilde{C}_{2}, \quad u_{1} \in \mathfrak{B}_{v}, \quad u_{2} \in E, t \in[0, T] .
$$

(B3) There exists a constant $\widetilde{C}_{3}>0$ such that

$$
\mathbb{E}\left\|H\left(t, u_{1}, u_{2}\right)\right\|^{2} \leq \widetilde{C}_{3}, \quad u_{1} \in \mathfrak{B}_{v}, \quad u_{2} \in E, t \in[0, T] .
$$

Theorem 4.1. Let us suppose that assumptions of Theorem 3.1 hold and (B1)(B3) are fulfilled and the linear system corresponding to system (1.1)-(1.3) is approximately controllable on $[0, T]$. Then, stochastic control system (1.1) involving fractional derivative is approximately controllable on $[0, T]$.

Proof. Let $u^{\lambda}(\cdot)$ be a fixed point of $\Psi$ in $\mathfrak{B}_{T}$. Theorem 3.1 gives that any fixed point of the operator $\Psi$ is the mild solution of the system (1.1)-(1.3). By using the stochastic Fubini theorem, any fixed point of $\Psi$ is a mild solution of (1.1) if $u^{\lambda}(t)$ fulfills

$$
u^{\lambda}(T)=u_{T}-\lambda R\left(\lambda, \Gamma_{0}^{T}\right) k\left(u^{\lambda}(\cdot)\right)
$$

where

$$
\begin{aligned}
k\left(u^{\lambda}(\cdot)\right)= & \mathbb{E} u_{T}+\int_{0}^{T} \sigma(s) d W(s)-S_{q}(t)\left(\phi(0)-g\left(u^{\lambda}\right)\right)-K_{q}(t)\left(u_{1}-h\left(u^{\lambda}\right)\right) \\
& -\sum_{i=1}^{\infty} S_{q}\left(T-t_{i}\right) I_{i}\left(u^{\lambda}\left(t_{i}\right)\right)-\sum_{t_{i}<t} K_{q}\left(T-t_{i}\right) J_{i}\left(u^{\lambda}\left(t_{i}\right)\right)+\int_{0}^{T} K_{q}(T-s) \\
& \times G\left(s, u_{s}^{\lambda}, \int_{0}^{s} a_{1}\left(s, \zeta, u_{\zeta}^{\lambda}\right) d \zeta\right) d s \\
& -\int_{0}^{T} R_{q}(T-s) F\left(s, u_{s}^{\lambda}, \int_{0}^{s} a_{2}\left(s, \zeta, u_{\zeta}^{\lambda}\right) d \zeta\right) d s \\
& -\int_{0}^{T} R_{q}(T-s) H\left(s, u_{s}^{\lambda}, \int_{0}^{s} a_{3}\left(s, \zeta, u_{\zeta}^{\lambda}\right) d \zeta\right) d W(s)
\end{aligned}
$$

By the assumptions (B1)-(B3), we have that $F, G$ and $H$ are uniformly bounded on $[0, T]$. Then there are subsequence, denoted by $\left\{G\left(s, u_{s}^{\lambda}, \int_{0}^{s} a_{1}\left(s, \zeta, u_{\zeta}^{\lambda}\right) d \zeta\right)\right\}$, 
$\left\{F\left(s, u_{s}^{\lambda}, \int_{0}^{s} a_{2}\left(s, \zeta, u_{\zeta}^{\lambda}\right) d \zeta\right)\right\}$ and $\left\{H\left(s, u_{s}^{\lambda}, \int_{0}^{s} a_{3}\left(s, \zeta, u_{\zeta}^{\lambda}\right) d \zeta\right)\right\}$ which converges weakly to say $G(s), F(s)$ and $H(s)$ in $E, E$ and $\mathcal{L}(K, E)$, respectively. On the other hand, the operator $\lambda\left(\lambda I+\Gamma_{s}^{T}\right)^{-1}$ strongly as $\lambda \rightarrow 0^{+}$for all $s \in[0, T]$. Thus, by Lebesgue dominated convergence theorem, we have that for $t \in[0, T]$,

$$
\begin{aligned}
& \left\|u^{\lambda}(T)-u_{T}\right\|^{2} \leq 6 \mathbb{E} \| \lambda R\left(\lambda, \Gamma_{0}^{T}\right)\left[\mathbb{E} u_{T}\right. \\
& \left.+\int_{0}^{T} \sigma(s) d W(s)-S_{q}(t)\left[\phi(0)-g\left(u^{\lambda}\right)\right]-K_{q}(s)\left(u_{1}-h\left(u^{\lambda}\right)\right)\right] \| \\
& +6 \mathbb{E}\left\|\sum_{i=1}^{\infty} \lambda R\left(\lambda, \Gamma_{0}^{T}\right) S_{q}\left(T-t_{i}\right) I_{i}\left(u^{\lambda}\left(t_{i}\right)\right)\right\|^{2} \\
& +6 \mathbb{E}\left\|\sum_{t_{i}<t} \lambda R\left(\lambda, \Gamma_{0}^{T}\right) K_{q}\left(T-t_{i}\right) J_{i}\left(u^{\lambda}\left(t_{i}\right)\right)\right\|^{2} \\
& +6 \mathbb{E}\left\|\int_{0}^{T} \lambda R\left(\lambda, \Gamma_{0}^{T}\right) K_{q}(T-s) \times\left[G\left(s, u_{s}^{\lambda}, \int_{0}^{s} a_{1}\left(s, \zeta, u_{\zeta}^{\lambda}\right) d \zeta\right)-G(s)\right] d s\right\|^{2} \\
& +6 \mathbb{E}\left\|\int_{0}^{T} \lambda R\left(\lambda, \Gamma_{0}^{T}\right) R_{q}(T-s) \times\left[F\left(s, u_{s}^{\lambda}, \int_{0}^{s} a_{2}\left(s, \zeta, u_{\zeta}^{\lambda}\right) d \zeta\right)-F(s)\right] d s\right\|^{2} \\
& +6 \mathbb{E}\left\|\int_{0}^{T} \lambda R\left(\lambda, \Gamma_{0}^{T}\right) R_{q}(T-s) \times\left[H\left(s, u_{s}^{\lambda}, \int_{0}^{s} a_{3}\left(s, \zeta, u_{\zeta}^{\lambda}\right) d \zeta\right)-H(s)\right] d w(s)\right\|^{2} \\
& \rightarrow 0, \text { as } \lambda \rightarrow 0 .
\end{aligned}
$$

This gives the approximate controllability of (1.1).

\section{EXAMPLE}

Consider an impulsive neutral stochastic partial differential equation with nonlocal conditions

$$
\begin{aligned}
& { }^{C} D_{t}^{q}\left[y(t, w)-\int_{0}^{t} \int_{-\infty}^{s}(t-s) e^{4(\tau-s)} y(\tau, w) d \tau d s-\int_{0}^{t}(t-s)\right. \\
& \left.\times \int_{0}^{s} \int_{-\infty}^{0} b_{1}\left(\tau_{1}\right) b_{2}\left(\tau_{2}\right) d \tau_{1} d \tau_{2} d s\right]=\frac{\partial^{2} y(t, w)}{\partial w^{2}}+\mu(t, w)+\int_{-\infty}^{0} \widetilde{a}_{1}(t, s, w, y(s, w)) d s \\
& +\int_{0}^{t} \int_{-\infty}^{0} \widetilde{a}_{2}(t) \widetilde{a}_{3}(s, \tau, w, y(s, w)) d \tau d s+\left(\int_{-\infty}^{0} \widetilde{c}_{1}(t, s, w, y(s, w)) d s\right. \\
& \left.+\int_{0}^{t} \int_{-\infty}^{0} \widetilde{c}_{2}(t) \widetilde{c}_{3}(s, \tau, w, y(s, w)) d \tau d s\right) \frac{d W(t)}{d t}, 0 \leq t \leq T, w \in[0, \pi] \\
& y(t, 0)=y(t, \pi)=0, \quad y^{\prime}(t, 0)=y^{\prime}(t, \pi)=0 \\
& y(0, w)+\int_{0}^{\pi} k_{1}(w, z) y(t, z) d z=\phi(t, w), \quad t \in(-\infty, 0]
\end{aligned}
$$




$$
\begin{aligned}
& y^{\prime}(0, w)+\int_{0}^{\pi} k_{2}(w, z) y(t, z) d z=\psi(t, w) \\
& \left.\Delta y(t, w)\right|_{t=t_{i}}=I_{i}\left(y\left(t_{i}^{-}, w\right)\right)=\int_{-\infty}^{0} \widetilde{d}_{i}(t-s) y(\theta, w) d s \\
& \left.\Delta y^{\prime}(t, w)\right|_{t=t_{i}}=J_{i}(y(t, w))=\int_{-\infty}^{0} \widetilde{f}_{i}(t-s) y(\theta, w) d s
\end{aligned}
$$

where $I_{i}, J_{i} \in C(\mathbb{R}, \mathbb{R}), i=1, \cdots, m, W(t)$ denotes a standard cylindrical Wiener process in $E$ defined on a stochastic space $(\Omega, \mathcal{F}, P)$ and $E=K=L^{2}([0, \pi])$ with the norm $\|\cdot\|, \mu:[0, T] \times[0, \pi] \rightarrow[0, \pi]$ is continuous in $t,{ }^{C} D_{t}^{q}$ represents the generalized Caputo fractional derivative of order $1<q<2$.

Choose $\mathbb{U}=E=L^{2}([0, \pi])$. Define the operator $A: D(A) \subset E \rightarrow E$ by $y^{\prime \prime}=A y$ with the domain

$$
D(A)=\left\{y \in E: y, y^{\prime} \text { are absolutely continuous, } y^{\prime \prime} \in E \text { and } y(0)=y(\pi)=0\right\} .
$$

Then, we have that $A$ is densely defined in $E$ and it is the infinitesimal generator of a resolvent family $\left\{S_{q}(t): t \geq 0\right\}$. Further, $A$ has a discrete spectrum with eigenvalues of the form $-n^{2}, n=0,1,2, \cdots$ and corresponding normalized eigenfunctions are given by $y_{n}(w)=\sqrt{\frac{2}{\pi}} \sin (n w)$. Additionally, $\left\{y_{n}: n \in \mathbb{N}\right\}$ is an orthonormal basis for $E$ and

$$
T(t) y=\sum_{i=1}^{\infty} e^{-n^{2} t}\left(y, y_{n}\right) y_{n}, \quad \forall \quad y \in E, t>0 .
$$

Now, we take $v(t)=e^{2 t}, t<0$. Then we have $l=\int_{-\infty}^{0} v(s) d s=1 / 2($ here $s<0)$ and define

$$
\|y\|_{\mathfrak{B}_{v}}=\int_{-\infty}^{0} v(s) \sup _{\theta \in[s, 0]}\left(\mathbb{E}\|y(\theta)\|^{2}\right)^{1 / 2} d s .
$$

Clearly, $\left(\mathfrak{B}_{v},\|\cdot\|_{\mathfrak{B}_{v}}\right)$ is a Banach space. Thus, for $(t, y) \in[0, T] \times \mathfrak{B}_{v}$ with $y(\theta)(w)=$ $y(\theta, w), \quad(\theta, w) \in(-\infty, 0] \times[0, \pi]$. Let $y(t) w=y(t, w)$ and define the bounded linear operator $B: \mathbb{U} \rightarrow E$ by $B x(t)(w)=\mu(t, w), w \in[0, \pi], u \in \mathbb{U}$. Thus, the functions $G:[0, T] \times \mathfrak{B}_{v} \times E \rightarrow E, F:[0, T] \times \mathfrak{B}_{v} \times E \rightarrow E$ and $H:[0, T] \times \mathfrak{B}_{v} \times E \rightarrow L_{\mathcal{Q}}(K, E)$ are given as

$$
\begin{aligned}
G\left(t, \phi, \int_{0}^{t} a_{1}(t, s, \phi) d s\right)(w) & =\int_{-\infty}^{t} e^{4(\tau-t)} \phi(\tau, w) d \tau \\
& +\int_{0}^{t} \int_{-\infty}^{0} b_{1}(t) b_{2}(\tau) \phi(\tau, w) d \tau d s, \\
F\left(t, \phi, \int_{0}^{t} a_{2}(t, s, \phi) d s\right)(w)=\int_{-\infty}^{0} \widetilde{a}_{1}(t, s, w, \phi(s, w)) d s & +\int_{0}^{t} \int_{-\infty}^{0} \widetilde{a}_{2}(t) \widetilde{a}_{3}(s, \tau, w, \phi(s, w)) d \tau d s,
\end{aligned}
$$




$$
\begin{aligned}
H\left(t, \phi, \int_{0}^{t} a_{3}(t, s, \phi) d s\right)(w)=\int_{-\infty}^{0} \widetilde{c}_{1}(t, s, w, \phi(s, w)) d s & \\
& +\int_{0}^{t} \int_{-\infty}^{0} \widetilde{c}_{2}(t) \widetilde{c}_{3}(s, \tau, w, \phi(s, w)) d \tau d s
\end{aligned}
$$

where:

(1) $b_{1}, b_{2}: \mathbb{R} \rightarrow \mathbb{R}$ are continuous, and

$$
\gamma_{2}=\left(\int_{-\infty}^{0} \frac{\left(b_{2}(s)\right)^{2}}{v(s)} d s\right)^{1 / 2}<\infty .
$$

(2) The functions $\widetilde{a}_{2}, \widetilde{c}_{2}: \mathbb{R} \rightarrow \mathbb{R}$ are continuous and $\widetilde{a}_{j}, \widetilde{c}_{j}(j=1,3): \mathbb{R} \rightarrow \mathbb{R}$ are continuous and there exist continuous functions $p_{i}, q_{i}: \mathbb{R} \rightarrow \mathbb{R}(i=1,2,3,4)$ such that

$$
\begin{aligned}
& \left|\widetilde{a}_{1}(t, s, x, y)\right| \leq p_{1}(t) p_{2}(s)|y|, \quad(t, s, x, y) \in \mathbb{R}^{4} \\
& \left|\widetilde{a}_{3}(t, s, x, y)\right| \leq p_{3}(t) p_{4}(s)|y|, \quad(t, s, x, y) \in \mathbb{R}^{4} \\
& \left|\widetilde{c}_{1}(t, s, x, y)\right| \leq q_{1}(t) q_{2}(s)|y|, \quad(t, s, x, y) \in \mathbb{R}^{4} \\
& \left|\widetilde{c}_{3}(t, s, x, y)\right| \leq q_{3}(t) q_{4}(s)|y|, \quad(t, s, x, y) \in \mathbb{R}^{4}
\end{aligned}
$$

with

$$
\begin{aligned}
L_{1}^{\widetilde{a}} & =\left(\int_{-\infty}^{0} \frac{\left(p_{2}(s)\right)^{2}}{v(s)} d s\right)^{1 / 2}<\infty, \quad L_{2}^{\widetilde{a}}=\left(\int_{-\infty}^{0} \frac{\left(p_{4}(s)\right)^{2}}{v(s)} d s\right)^{1 / 2}<\infty, \\
L_{1}^{\widetilde{c}} & =\left(\int_{-\infty}^{0} \frac{\left(q_{2}(s)\right)^{2}}{v(s)} d s\right)^{1 / 2}<\infty \text { and } L_{2}^{\widetilde{c}}=\left(\int_{-\infty}^{0} \frac{\left(q_{4}(s)\right)^{2}}{v(s)} d s\right)^{1 / 2}<\infty .
\end{aligned}
$$

(3) The functions $\widetilde{d}_{i}, \widetilde{f}_{i}$ and $L_{I_{i}}=\left(\int_{-\infty}^{0} \frac{\left(\widetilde{d}_{i}\right)^{2}}{v(s)} d s\right)^{1 / 2}, L_{J_{i}}=\left(\int_{-\infty}^{0} \frac{\left(\widetilde{f}_{i}\right)^{2}}{v(s)} d s\right)^{1 / 2}$, where $i=1, \cdots, m, m \in \mathbb{N}$ are finite.

Thus, the system (5.1)-(5.6) can be reformulated as (1.1)-(1.3) and neutral fractional stochastic system with nonlocal and impulsive conditions corresponding to (5.1)-(5.6) is approximately controllable. Therefore, we may easily verify all the assumptions of Theorem 3.1, 4.1 and hence, fractional control system (5.1)-(5.6) is approximately controllable on $(-\infty, T]$.

\section{ACKNOWLEDGMENT}

The authors would like to thank the referee for valuable comments and suggestions. The work of the first author is supported by IPDF, Indian Institute of Technology, Guwahati. 


\section{REFERENCES}

[1] A. Pazy, Semigroups of linear operators and aplications to partial differential equations, Springer, New York, 1983.

[2] A. A. Kilbas, H. M. Srivastava, J. J. Trujillo, Theory and Applications of Fractional Differential Equations, Elsevier, Amsterdam, 2006.

[3] I. Podlubny, Fractional differential equations, Mathematics in Science and Engineering, vol. 198. Academic Press, San Diego, 1999.

[4] J. K. Hale, J. Kato, Phase space for retarded equations with infinite delay, Funkcial. Ekvac., 21 (1978), 11-41.

[5] Y. Hino, S. Murakami, T. Naito, Functional Differential Equations with Infinite Delay, in Lecture Notes in Math., vol. 1473, Springer-Verlag, Berlin, 1991.

[6] V. Lakshmikantham, D. Baǐnov, P. S. Simeonov, Theory of impulsive differential equations, Series in Modern Applied Mathematics, World Scientific Publishing Co., Inc., Teaneck, NJ, 1989.

[7] M. Benchohra, J. Henderson, S. K. Ntouyas, Impulsive differential equations and inclusions, Contemporary Mathematics and Its Applications, Vol.2, Hindawi Publishing Corporation, New York, 2006.

[8] G. Da Prato, J. Zabczyk, Stochastic Equations in Infinite Dimensions, Cambridge University Press, Cambridge, 1992.

[9] B. Oksendal, Stochastic Differential Equations. Springer, Berlin, Germany, 5th edition, 2002.

[10] X. Mao, Stochastic Differential Equations and Applications, Woodhead Publishing, 2008.

[11] L. Byszewski, Theorems about the existence and uniqueness of solutions of a semilinear evolution nonlocal Cauchy problem, J. Math. Anal. Appl., 162 (1991), 497-505.

[12] L. Byszewski, V. Lakshmikantham, Theorem about the existence and uniqueness of a solution of a nonlocal abstract Cauchy problem in a Banach space, Applied Analysis, 40 (1990), 11-19.

[13] J. Wang, M. Fečkan, Y. Zhou, On the new concept of solutions and existence results for impulsive fractional evolution equations, Dynamics of PDE, 8 (2011), 345-361.

[14] X. Zhang, C. Zhu, C. Yuan, Approximate controllability of impulsive fractional stochastic differential equations with state-dependent delay, Adv. Diff. Equ., 2015 (2015), pp-12. 
[15] R. Sakthivel, Y. Ren, H. Kim, Asymptotic stability of second-order neutral stochastic differential equations, J. Math. Phy., 51 (2010), pp-9.

[16] R. Sakthivel, R. Ganesh, S.Suganya, Approximate controllability of fractional neutral stochastic system with infinite delay, Repo. Math. Phy., 70 (2012), 291311.

[17] X. Zhang, Chuanxi Zhu, C. Yuan, Approximate controllability of fractional impulsive evolution systems involving nonlocal initial conditions, Adv. Diff. Equ., 2015 (2015), pp-14.

[18] Zhenhai Liu, Xiuwen Li, On the controllability of impulsive fractional evolution inclusions in Banach spaces, J Optim Theory Appl, 156 (2013), 167-182.

[19] Yanchao Zang, Junping Li, Approximate controllability of fractional impulsive neutral stochastic differential equations with nonlocal conditions, Boundary Value Prob. 2013 (2013), pp-13.

[20] P. Balasubramaniam, V. Vembarasan, T. Senthilkumar, Approximate controallability of impulsive fractional integro-differential systems with nonlocal conditions in Hilbert space, Numer. Funct. Anal. Optimi., 35 (2014), 177-197.

[21] C. Ravichandran, J. J. Trujillo, Controllability of impulsive fractional functional integro-differential equations in Banach spaces, J. Funct. Spaces Appl., 2013 (2013), Art. ID 812501, pp-8.

[22] Z. Yan, H. Zhang, Asymptotic stability of fractional impulsive neutral stochastic partial integro-differential equations with infinite delay, Elect. J. Diff. Equ., 2013 (2013), No-206, page 29.

[23] X. Zhang, C. Zhu, C. Yuan, Approximate controllability of impulsive fractional stochastic differential equations with state-dependent delay, Adv. Diff. Equ., 2015 (2015), pp-12.

[24] R. Sakthivel, Y. Ren, Approximate controllability of fractional differential equations with state-dependent delay, Results. Math., 63 (2015), 949-963.

[25] E. Bazhlekova, Fractional evolution equations in Banach spaces, Ph.D. Thesis, Eindhoven University of Technology, 2001.

[26] R. Agarwal, M. Meehan, D. O'Regan, Fixed point theory and applications, in: Cambridge Tracts in Mathematics, Cambridge University Press, New York, 2001, pp-178-179.

[27] X.-B. Shu, Y. Lai, Y. Chen, The existence of mild solutions for impulsive fractional partial differential equations, Nonlinear Analysis: TMA, 74 (2011), 20032011.

[28] R. Sakthivel, P. Revathi, Y. Ren, Existence of solutions for nonlinear fractional stochastic differential equations, Nonlinear Analysis: TMA, 81 (2013), 70-86. 
[29] Y. Li, B. Liu, Existence of solution of nonlinear neutral functional differential inclusions with infinte delay, Stoch. Anal. Appl., 25 (2007), 397-415.

[30] M. Johannes, The statistical and economic role of jumps in continuous-time interest rate models, J. Finance, 59 (2004), 227260.

[31] R. Curtain, H. J. Zwart, An introduction to infinite dimensional linear systems theory. New York: Springer-Verlag, 1995.

[32] N. I. Mahmudov, S. Zorlu, On the approximate controllability of fractional evolution equations with compact analytic semigroup, J. Comput. Appl. Math., 259 (2014), 194-204.

[33] R. Sakthivel, R. Ganesh, Y. Ren, S. M. Anthoni, Approximate controllability of nonlinear fractional dynamical systems, Commun. Nonlinear Sci. Numer. Simul., 18 (2013), 3498-3508.

[34] R. Sakthivel, S. Suganya, S. M. Anthoni, Approximate controllability of fractional stochastic evolution equations, Comput. Math. Appl., 63 (2012), 660-668.

[35] R. Ganesh, R. Sakthivel, N. I. Mahmudov, S. M. Anthoni, Approximate controllability of fractional integro-differential evolution equations, J. Appl. Math., 2013, Art. ID 291816.

[36] T. Guendouzi, S. Farahi, Approximate controllability of semilinear fractional stochastic dynamic systems with nonlocal conditions in Hilbert spaces, Mediterr. J. Math., 13 (2016), 637-656.

[37] X.-B. Shu, Q. Wang, The existence and uniqueness of mild solutions for fractional differential equations with nonlocal conditions of order $q \in(1,2)$, Comp. Math. Appl., 64 (2012) 2100-2110.

[38] X.-B. Shu, Y. Shi, A study on the mild solution of impulsive fractional evolution equations, Appl. Math. Comp., 273 (2016), 465-476.

[39] P. Muthukumar, K. Thiagu, Existence of solutions and approximate controllability of fractional nonlocal neutral impulsive stochastic differential equations of order $1<q<2$ with infinite delay and Poisson jumps, J. Dyn. Control Syst., 2015, pp-23.

[40] X. Wang, X.-B. Shu, The existence of positive mild solutions for fractional differential evolution equations with nonlocal conditions of order $1<\alpha<2$, Adv. Diff. Equ., 2015 (2015), pp-15.

[41] T. Taniguchi, Kai Liu, A. Truman, Existence, uniqueness and asymptotic behavior of mild solutions to stochastic functional differential equations in Hilbert spaces, J. Diff. Equ., 181 (2002), 72-91.

[42] L. Pan, Existence of the mild solution for impulsive stochastic differential equations with nonlocal conditions, Diff. Equ. Appl., 4 (2012), 485-494. 
[43] A. Chadha, D. N. Pandey, Existence results for an impulsive neutral stochastic fractional integro-differential equation with infinite delay, Nonlinear Analysis, 128 (2015) 149-175.

[44] A. Chadha, D. N. Pandey, FaedoGalerkin approximation of solution for a nonlocal neutral fractional differential equation with deviating argument, Mediterr. J. Math., 13 (2016), 3041-3067.

[45] A. Chadha, D. N. Pandey, Existence results for an impulsive neutral fractional integrodifferential equation with infinite delay, Int. J. Diff. Equ., 2014 (2014), pp-10.

[46] R. Murugesu, V. Vijayakumar, J. P. C. dos Santos, Existence of the mild solutions for nonlocal Cauchy problem for fractional neutral integro-differential equations with unbounded delay, Commun. Math. Anal. 14 (2013), 59-71.

[47] V.B. Kolmanovsky, V.R. Nosov, Stability of neutral-type functional differential equations, Nonlinear Analysis: TMA, 6 (1982), 873-910.

[48] Zhang Yong, On the uniqueness of solutions of neutral functional differential equations, J. Math. Anal. Appl., 161 (1991), 426-439.

[49] P. Kalamani, D. Baleanu, S. Selvarasu, M. M. Arjunan, On existence results for impulsive fractional neutral stochastic integro-differential equations with nonlocal and state-dependent delay conditions, Adv. Diff. Equ., 2016 (2016), pp-36.

[50] R. Sakthivel, Yong Ren, A. Debbouche, N.I. Mahmudov, Approximate controllability of fractional stochastic differential inclusions with nonlocal conditions, Applicable Analysis, 2016, pp-22.

[51] A. Debbouche, Delfim FM Torres, Approximate controllability of fractional nonlocal delay semilinear systems in Hilbert spaces, Int. J. Cont., 86 (2013), 15771585 .

[52] M. Kerboua, A. Debbouche, D. Baleanu, Approximate controllability of Sobolev type nonlocal fractional stochastic dynamic systems in Hilbert spaces, Abst. Appl. Anal., 2013 (2013), pp-10. 
